\title{
Al'tshuler-Aronov-Spivak oscillations of bosonic matter-wave beams in the presence of interaction
}

\author{
Renaud Chrétien, ${ }^{1, *}$ Josef Rammensee, ${ }^{2}$ Julien Dujardin, ${ }^{1}$ Cyril Petitjean, ${ }^{1}$ and Peter Schlagheck ${ }^{1}$ \\ ${ }^{1}$ CESAM Research Unit, University of Liège, 4000 Liège, Belgium \\ ${ }^{2}$ Institut für Theoretische Physik, Universität Regensburg, 93040 Regensburg, Germany
}

(Received 21 December 2018; published 5 September 2019)

\begin{abstract}
We study theoretically the propagation of a guided atom laser across an Aharonov-Bohm ring which is exposed to a synthetic gauge field. The presence of disorder within the ring gives rise to Al'tshuler-AronovSpivak oscillations, seen in the disorder average of the transmission as a function of the effective gauge flux that is contained within the ring. Those oscillations are induced by coherent backscattering and represent a manifestation of weak localization. Through analytical and numerical calculations that are based on the meanfield Gross-Pitaevskii approximation for the propagating Bose-Einstein condensate, we show that the presence of a very weak atom-atom interaction within the ring leads to an inversion of the Al'tshuler-Aronov-Spivak oscillations, in a very similar manner as for the coherent backscattering of Bose-Einstein condensates within two-dimensional disorder potentials. Numerical simulations based on the truncated Wigner method reveal that this signature of weak antilocalization becomes washed out if the interaction strength is increased.
\end{abstract}

DOI: 10.1103/PhysRevA.100.033606

\section{INTRODUCTION}

Weak localization [1,2] is a physical effect related to a notable increase of the reflection of coherent waves traversing a disordered scattering region, compared with the incoherent transport process. As a result of current conservation, this increase is responsible for a drop in the transmission. This effect has been studied in mesoscopic physics for a long time now, as it highlights a macroscopic outcome resulting from quantum interferences. In solid-state physics, positive corrections to the resistivity of a disordered sample, due to weak localization, must indeed be added to classical predictions of the Drude formula $[3,4]$ describing electronic transport through a disordered sample [5]. They originate from a constructive interference between the contributions resulting from a scattering path and its time-reversal counterpart, which survives the disorder average.

A most prominent signature of quantum interference in mesoscopic physics is coherent backscattering [6-8]. This phenomenon, which is encountered in a wide variety of domains, is responsible for an enhancement of the backscattered current of a disordered sample that is illuminated by coherent waves, involving exactly the same mechanism as weak localization. The effect of coherent backscattering was already detected in 1893, namely with the observation that Saturn's rings are twice brighter in the backscattered direction [9]. More recently, coherent backscattering was observed in laboratory by illuminating a powder with laser light $[6,10]$, but also for acoustic waves [11] and elastic waves [12]. It is also used in seismology to probe the underground deeply and for the research of oil [13].

Most recently, coherent backscattering was also studied with matter waves by means of Bose-Einstein condensates

\footnotetext{
*rchretien@ulg.ac.be
}

[14]. In this context, new questions related to many-body physics arise, especially concerning the interplay between quantum interferences and the presence of interaction. In a quasistationary context, mean-field studies [15-17] show that the presence of a nonlinearity in the wave equation describing the transport of ultracold bosonic atoms across a disordered region can give rise to an inversion of the coherent backscattering peak. On the other hand, many-body diagrammatic approaches [18] indicate that this inversion should be limited to a mean-field regime of very low atom-atom interaction strengths, while in a more realistic situation a dephasing is to be expected.

To shed more light on this issue, we propose to verify these observations in a most elementary setting which allows for numerical simulations beyond the mean-field Gross-Pitaevskii approach. Our system consists of two leads connected to a ring-shaped resonator that is threaded by a synthetic gauge flux. For such a system, it is well known that AharonovBohm oscillations [19-22] take place in the transmission. The presence of disorder within the ring is further responsible for a crossover from Aharonov-Bohm to Al'tshuler-Aronov-Spivak oscillations [22-25], which is a mesoscopic phenomenon related to weak localization. Al'tshuler-Aronov-Spivak oscillations have been investigated in some detail in mesoscopic physics (see, e.g., Refs. [26-29]) and the effects of electronelectron interaction were discussed [30].

In this paper we investigate how Al'tshuler-Aronov-Spivak oscillations behave in the presence of bosonic interaction. To this end, we compute the disorder-averaged transmission of an interacting guided-atom-laser beam across a ring by means of a numerical integration of the Gross-Pitaevskii equation and compare its findings with the predictions of a nonlinear diagrammatic theory. An inversion of the Al'tshuler-Aronov-Spivak oscillations profile in the disorderaveraged transmission is indeed encountered at small nonlinearities, in analogy with previous studies [15-17] on coherent 
backscattering. Finally, we use the truncated Wigner method, which allows one to go beyond the mean-field approximation in order to verify the extent to which this phenomenon prevails in the presence of finite atom-atom interaction strengths.

We start by presenting in Sec. II the guided-atom-laser configuration under study and the spatial discretization scheme that we use to numerically solve the equations that describe our system. By representing the ring and the leads appearing in our system as a quantum graph, we are in a position to formulate in Sec. III a theory explaining the appearance of Al'tshuler-Aronov-Spivak oscillations in the noninteracting case. We then present in Sec. IV the numerical methods we use, namely, the Gross-Pitaevskii equation and the truncated Wigner method. In Sec. V we first apply these methods to study Aharonov-Bohm oscillations in the presence of interaction. We then discuss the transition from Aharonov-Bohm to Al'tshuler-Aronov-Spivak oscillations and investigate how the latter are affected by the presence of interaction. Numerical findings on disorder-averaged transmission are compared with predictions of nonlinear diagrammatic theory, which is described in Sec. VI, following the scheme developed in [31].

\section{DESCRIPTION OF THE SYSTEM}

The system we study is a Bose-Einstein condensate of $\mathcal{N} \rightarrow \infty$ particles at zero temperature $T=0$ and chemical potential $\mu$, which is outcoupled from a trap to a waveguide, e.g., by means of a radio-frequency knife [32-34] or a multiphoton Raman transition [35-37], following the principle of an atom laser [33,34,38-42]. Engineered in this waveguide is a two-arm ring, similar to an interferometer, in which a synthetic gauge field $[43,44]$ with tunable magnetic flux $\Phi$ is produced, the role of which is to break the symmetry between the two arms of the ring. Because of that flux, a different phase shift will be acquired by the atoms depending upon which arm is chosen to cross the ring. This symmetry breaking then gives rise to the Aharonov-Bohm interference effect [19-22] scenario. Experimentally, such rings can be obtained by the perpendicular intersection of red-detuned lasers, as is explained in Refs. [45,46]. A horizontal atomic waveguide in a particular direction can be engineered by using a far-detuned laser beam, as in Ref. [38]. The ring-shaped geometry would then be connected to two semi-infinite leads, as is represented in Fig. 1(a).

The model to describe this transport process is provided by a system of evolution equations for the field operator $\hat{\phi}_{S}(t)$ of the source and the field operators $\hat{\psi}(x, t)$ of atoms within the waveguide structure, with $x$ representing positions in the leads and the ring. For a single infinite lead, we would have [47]

$$
\begin{aligned}
i \hbar \frac{\partial \hat{\psi}(x, t)}{\partial t}= & \hat{H}_{0} \hat{\psi}(x, t)+g(x) \hat{\psi}^{\dagger}(x, t) \hat{\psi}(x, t) \hat{\psi}(x, t) \\
& +K(x, t) \hat{\phi}_{S}, \\
i \hbar \frac{\partial \hat{\phi}_{S}(t)}{\partial t}= & \mu \hat{\phi}_{S}+\int d x K^{*}(x, t) \hat{\psi}(x, t),
\end{aligned}
$$

with $K(x, t)$ the position-dependent coupling strength of the coupling between source and leads, $\mu$ the chemical potential of the source, and $g(x)$ the effective one-dimensional interaction strength (which we assume to be present only inside

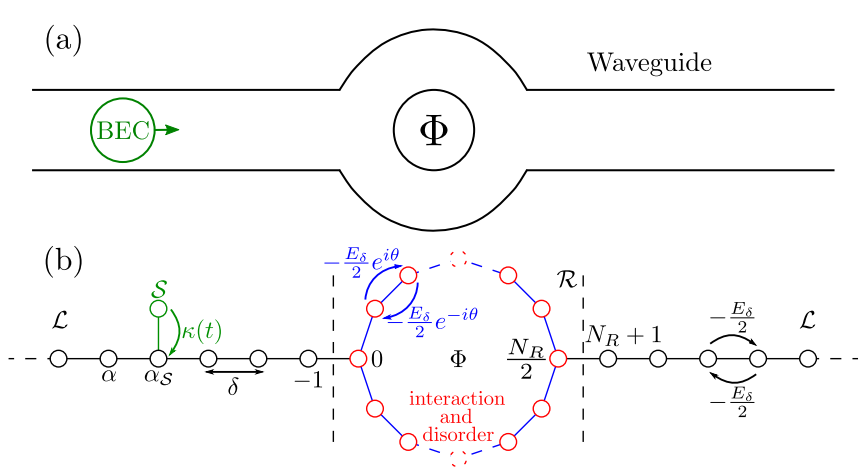

FIG. 1. (a) Sketch of the system under study. A Bose-Einstein condensate at temperature $T=0$ and chemical potential $\mu$ in a trap (sketched by a green circle) is outcoupled to a (semi-infinite) waveguide by, for instance, a radio-frequency knife or multiphoton Raman transition. This waveguide is connected to another semiinfinite waveguide via a two-arm ring. In this ring a tunable artificial gauge field $\Phi$ is induced. (b) Discretization of the infinite 1D space, which is artificially subdivided into several regions labeled $\mathcal{L}$ (leads), $\mathcal{R}$ (ring), and $\mathcal{S}$ (source). Sites depicted in red exhibit both interaction and disorder. Edges depicted in blue exhibit, in addition to the Bose-Hubbard hopping term $-E_{\delta} / 2$, a Peierls phase $[48,49]$ shift $e^{ \pm i \theta}$ depending on the direction of the rotation, due to an artificial gauge field. The phase shift $\theta$ acquired at each jump from one site to its neighbor is given by $\theta=\Phi / N_{R}$, with $N_{R}$ the number of sites within the ring.

the ring). The one-dimensional single-particle Hamiltonian (without the artificial gauge field) is given by

$$
\hat{H}_{0}=\hat{H}_{k}+V(x) \text {, }
$$

with $\hat{H}_{k}=-\frac{\hbar^{2}}{2 m} \frac{\partial^{2}}{\partial x^{2}}$ and $V(x)$ the disorder potential (which, like the interaction, is assumed to be present only inside the ring).

In view of implementing the truncated Wigner method, we discretize the one-dimensional space, as shown in Fig. 1(b), in a series of sites labeled by an index $\alpha$ and spaced by $\delta$. We describe the kinetic energy operator in terms of a finitedifference scheme

$$
\frac{\partial^{2}}{\partial x^{2}} \psi(x) \simeq \frac{\psi(x+\delta)+\psi(x-\delta)-2 \psi(x)}{\delta^{2}} .
$$

Through discretization, each site obtains an on-site energy $E_{\delta}=\hbar^{2} / m \delta^{2}$, with $\hbar$ the reduced Planck constant and $m$ the mass of the atoms, and a nearest-neighbor hopping $E_{\delta} / 2$.

We define several space regions in our discretization, namely, $\mathcal{L}$ for the leads, $\mathcal{R}$ for the ring, and $\mathcal{S}$ for the source. Smooth exterior complex scaling [50-57] is used according to Ref. [58] for absorption at both ends of the leads to avoid artifacts due to the finite extension of the leads in the numerical treatment. The effective Bose-Hubbard Hamiltonian describing this system can be decomposed into four subHamiltonians

$$
\hat{H}=\hat{H}_{\mathcal{L}}+\hat{H}_{\mathcal{L R}}+\hat{H}_{\mathcal{R}}+\hat{H}_{\mathcal{S}}
$$


with

$$
\begin{gathered}
\hat{H}_{\mathcal{L}}=\sum_{\alpha \in \mathcal{L}}\left[E_{\delta} \hat{a}_{\alpha}^{\dagger} \hat{a}_{\alpha}-\frac{E_{\delta}}{2}\left(\hat{a}_{\alpha+1}^{\dagger} \hat{a}_{\alpha}+\hat{a}_{\alpha}^{\dagger} \hat{a}_{\alpha+1}\right)\right], \\
\hat{H}_{\mathcal{L} \mathcal{R}}=-\frac{E_{\delta}}{2}\left(\hat{a}_{-1}^{\dagger} \hat{a}_{0}+\hat{a}_{0}^{\dagger} \hat{a}_{-1}+\hat{a}_{N_{R} / 2}^{\dagger} \hat{a}_{N_{R}+1}+\hat{a}_{N_{R}+1}^{\dagger} \hat{a}_{N_{R} / 2}\right), \\
\hat{H}_{\mathcal{R}}=\sum_{\alpha \in \mathcal{R}}\left[\left(E_{\delta}+V_{\alpha}\right) \hat{a}_{\alpha}^{\dagger} \hat{a}_{\alpha}-\frac{E_{\delta}}{2}\left(\hat{a}_{\alpha-1}^{\dagger} \hat{a}_{\alpha} e^{i \theta}\right.\right. \\
\left.\left.\quad+\hat{a}_{\alpha+1}^{\dagger} \hat{a}_{\alpha} e^{-i \theta}\right)+\frac{g}{2} \hat{a}_{\alpha}^{\dagger} \hat{a}_{\alpha}^{\dagger} \hat{a}_{\alpha} \hat{a}_{\alpha}\right] \\
\hat{H}_{\mathcal{S}}=\kappa(t) \hat{a}_{\alpha_{\mathcal{S}}}^{\dagger} \hat{b}+\kappa^{*}(t) \hat{b}^{\dagger} \hat{a}_{\alpha \mathcal{S}}+\mu \hat{b}^{\dagger} \hat{b},
\end{gathered}
$$

each of which is associated with the corresponding region of the space they are labeled by.

In this Hamiltonian, we have introduced by $\hat{a}_{\alpha}^{\dagger}$ and $\hat{a}_{\alpha}$ the creation and annihilation operators at site $\alpha$ and by $\hat{b}^{\dagger}$ and $\hat{b}$ the creation and annihilation operators of the source which is maintained at chemical potential $\mu$ and $T=0 .{ }^{1}$ We treat this source as a Bose-Einstein condensate containing $\mathcal{N} \rightarrow \infty$ atoms and make the approximation that it is connected to one single lattice site labeled by $\alpha_{\mathcal{S}}$. The coupling $\kappa(t)$ between the source and the leads is smoothly ramped on with time (for instance, by varying the intensity of the radio-frequency field in the case of a radio-frequency knife) and approaches a maximal value. The latter tends to zero such that $\mathcal{N}|\kappa(t)|^{2}$ remains constant $[38,59,60]$, which implies that the number of atoms in the scattering region remains constant too. In this limit, a stationary many-body scattering state can therefore be achieved. In the case where the source is connected, as described, to an infinite lead, which amounts to considering $\hat{H}=\hat{H}_{\mathcal{S}}+\hat{H}_{\mathcal{L}}$, it would inject a free flux of atoms yielding a stationary density and current given by $[58,60]$

$$
\begin{aligned}
\rho^{\varnothing} & =\frac{1}{\delta} \frac{\mathcal{N}|\kappa(t)|^{2}}{\mu\left(2 E_{\delta}-\mu\right)}, \\
j^{\varnothing} & =\frac{1}{\hbar} \frac{\mathcal{N}|\kappa(t)|^{2}}{\sqrt{\mu\left(2 E_{\delta}-\mu\right)}} .
\end{aligned}
$$

The on-site interaction strength is controlled by the parameter [61] $g=2 \hbar \omega_{\perp} a_{S} / \delta, \omega_{\perp}$ being the perpendicular confinement frequency of the trap and $a_{S}$ the $s$-wave scattering length. Finally, disorder is brought into the system through the on-site parameters $V_{\alpha}$. The disorder we use is in the continuous space generated by $[62,63]$

$$
V(x)=\bar{V}_{0} \int \frac{1}{\sqrt{\sigma \sqrt{\pi}}} \exp \left[-\frac{(x-y)^{2}}{2 \sigma^{2}}\right] \eta(y) d y,
$$

where $\bar{V}_{0}$ is the amplitude of the disorder and $\sigma$ its correlation length. The correlator $\eta(y)$ is a Gaussian random white noise with zero mean and unit variance, i.e., $\langle\eta(x) \eta(y)\rangle=\delta(x-y)$, with $\langle\cdot\rangle$ the random average. In the framework of the above

\footnotetext{
${ }^{1}$ Note that within the Hamiltonian $\hat{H}_{\mathcal{R}}$ we have identified the sites $\alpha_{N_{R}+1} \equiv \alpha_{0}$ and $\alpha_{-1} \equiv \alpha_{N_{R}}$ to simplify notation. They should not be confused with sites in the leads carrying the same labels.
}

discretization scheme, disorder is then represented by the onsite energies

$$
V_{\alpha}=\bar{V}_{0} \sum_{\alpha^{\prime}=0}^{N_{R}} \frac{1}{\sqrt{\sigma \sqrt{\pi}}} \exp \left[-\frac{\delta^{2}}{2 \sigma^{2}}\left(\alpha-\alpha^{\prime}\right)^{2}\right] \eta_{\alpha^{\prime}} .
$$

This imposes a condition on the discretization, namely, $\delta \ll$ $\sigma$, in order that the discretization scheme captures the details of the disorder.

\section{THEORY OF AL'TSHULER-ARONOV-SPIVAK OSCILLATIONS}

Let us first consider the noninteracting case, which corresponds to a ring penetrated by an Aharonov-Bohm artificial gauge flux in the presence of disorder but without any interaction. The ring and the leads can be represented as a quantum graph [64-66] with two vertices, two internal bonds of finite length, and two external bonds of infinite extension. On this graph, the Green's function can be represented as a sum over all possible paths $\gamma$ linking two given points $\alpha^{\prime}$ and $\alpha$ on the graph (see Appendix A),

$$
G\left(\alpha, \alpha^{\prime}, \mu\right)=\frac{1}{i E_{\delta} \sin (k \delta)} \sum_{\gamma} A_{\gamma} e^{i S_{\gamma} / \hbar},
$$

with $k \delta=\arccos \left(1-\mu / E_{\delta}\right) \simeq \sqrt{2 \mu / E_{\delta}}$ for $0<\mu / E_{\delta} \ll 1$. In Eq. (10), $S_{\gamma}$ is the accumulated action integral along the path $\gamma$ and the prefactor $A_{\gamma}=r^{n_{r}} t^{n_{t}}$ is the product of reflection and transmission matrix elements at each junction that a trajectory encounters, where $n_{r}\left(n_{t}\right)$ is the number of reflections (transmissions) along the path $\gamma$. Those matrix elements can be obtained from the analysis of a scattering problem across a symmetric $\mathrm{Y}$ junction, in the absence of disorder. Denoting by $E_{Y}$ the energy on the junction sites, which may be assumed to be different from the other on-site energies $E_{\delta}$, we obtain

$$
\begin{aligned}
& r=-\frac{1-E_{Y} / E_{\delta}+\frac{1}{2} e^{i k \delta}}{1-E_{Y} / E_{\delta}+e^{i k \delta}-\frac{1}{2} e^{-i k \delta}}, \\
& t=\frac{i \sin k \delta}{1-E_{Y} / E_{\delta}+e^{i k \delta}-\frac{1}{2} e^{-i k \delta}} .
\end{aligned}
$$

Those probability amplitudes satisfy the continuity and conservation of current equations

$$
\begin{aligned}
1+r & =t, \\
|r|^{2}+2|t|^{2} & =1 .
\end{aligned}
$$

In the continuous limit where the spacing $\delta \rightarrow 0$ vanishes, a nonvanishing transmission is obtained only for $E_{Y}=\frac{3}{2} E_{\delta}$, which yields

$$
\begin{aligned}
& r=-\frac{e^{i k \delta}-1}{2 e^{i k \delta}-1-e^{-i k \delta}} \stackrel{k \delta \rightarrow 0}{\longrightarrow}-\frac{1}{3}, \\
& t=\frac{e^{i k \delta}-e^{-i k \delta}}{2 e^{i k \delta}-1-e^{-i k \delta}} \stackrel{k \delta \rightarrow 0}{\longrightarrow} \frac{2}{3} .
\end{aligned}
$$

Here we choose $E_{Y}=E_{\delta}$, which corresponds to the case of a nearly closed ring (actually with nearly disconnected arms) that is weakly connected to the waveguides, as well as $\mu=$ 
$0.2 E_{\delta}$. This yields the following expressions for $r$ and $t$ :

$$
\begin{aligned}
& r=-\frac{e^{i k \delta}}{2 e^{i k \delta}-e^{-i k \delta}}=-\frac{43}{97}+\frac{24 i}{97}, \\
& t=\frac{e^{i k \delta}-e^{-i k \delta}}{2 e^{i k \delta}-e^{-i k \delta}}=\frac{54}{97}+\frac{24 i}{97} .
\end{aligned}
$$

The disorder within the ring is supposed to be weak and smooth, with an amplitude $\bar{V}_{0} \ll \mu$ and a spatial correlation length $\sigma$ satisfying $k \sigma \gg 1$ as well as $\sigma \gg \delta$ such that reflections inside each arm of the ring can safely be neglected. The action integral within each arm can then be written as

$$
\begin{aligned}
S & =\hbar \sum_{\alpha=1}^{N_{R}} \arccos \left(1-\frac{\mu-V_{\alpha}}{E_{\delta}}\right) \simeq \hbar \int_{0}^{N_{R}} \sqrt{\frac{\mu-V_{\alpha}}{2 E_{\delta}}} d \alpha \\
& \simeq N_{R} \hbar \sqrt{\frac{\mu}{E_{\delta}}}-\frac{\hbar}{2 \sqrt{\mu E_{\delta}}} \int_{0}^{N_{R}} V_{\alpha} d \alpha
\end{aligned}
$$

which means that the phase factors for the upper branch $\exp \left(i S_{u} / \hbar\right)$ and the lower branch $\exp \left(i S_{d} / \hbar\right)$ can safely be considered as independent complex random numbers with unit norm, provided the ring is sufficiently long such that

$$
\overline{\left|\int_{0}^{N_{R}} V_{\alpha} d \alpha\right|} \gg \pi \sqrt{\mu E_{\delta}} .
$$

Now if we consider the presence of an Aharonov-Bohm flux $\Phi$ within the ring, we can write

$$
\exp \left(\frac{i}{\hbar} S_{u}\right)=\exp \left[i\left(\Phi_{u} \pm \frac{\Phi}{2}\right)\right]
$$

and

$$
\exp \left(\frac{i}{\hbar} S_{d}\right)=\exp \left[i\left(\Phi_{d} \mp \frac{\Phi}{2}\right)\right]
$$

for the upper and the lower arm, respectively, where the upper (lower) sign is associated with the path from the left to the right (the right to the left) junction and $\Phi_{u}$ and $\Phi_{d}$ are random phases accounting for disorder in the upper or lower arms of the ring.

Reflection and transmission amplitudes across the ring can then be obtained from the Green's function $G\left(\alpha, \alpha_{\mathcal{S}}, \mu\right)$, with $\alpha$ being located in the lead before or behind the ring for reflection and transmission, respectively. More precisely, these amplitudes are obtained by normalizing these Green's functions with respect to the Green's function of free motion along a clean one-dimensional (1D) lattice, namely,

$$
G_{0}=\frac{e^{i k\left(\alpha-\alpha_{\mathcal{S}}\right)}}{i E_{\delta} \sin (k \delta)} .
$$

The probability amplitude of reflection $R$ can therefore be written as a sum of probability amplitudes associated with trajectories yielding a reflection after a possible complicated journey in the ring. At each $Y$ junction, such an amplitude is multiplied either by $r$ in the case of reflection or by $t$ in the case of transmission across this junction. In addition, it is also multiplied by the phase factor accounting for its journey within the ring. Each exploration of the upper (lower) branch yields an $\exp \left(i \Phi_{u}\right)\left[\exp \left(i \Phi_{d}\right)\right]$ phase factor and each trip in the

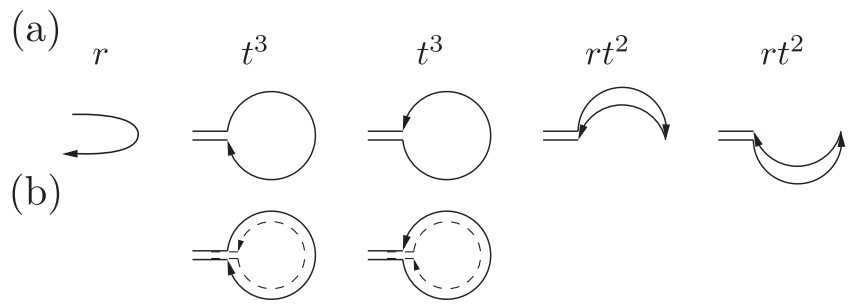

FIG. 2. (a) Schematic representation of the diagrams yielding an expression for the reflection up to corrections of power 5 in $r$ and $t$, as is shown in Eq. (24). Since at each junction either a reflection or a transmission event occurs, we have to multiply each trajectory amplitude by either $r$ or $t$, depending on which event took place. An additional phase factor $\exp \left[i\left(\Phi_{u / d} \pm \Phi\right)\right]$ should also be considered as a result of the exploration of the upper or lower branch in the clockwise or counterclockwise direction of rotation. (b) Sketch of the intensity diagrams that give rise to the leading-order terms beyond the diagonal approximation. The two paths corresponding to the wave function (solid line) and its complex conjugate (dashed line) interfere constructively (destructively) for fluxes $\Phi$ that are an even (odd) multiple of $\pi / 2$.

clockwise (counterclockwise) direction yields an $e^{i \Phi}\left(e^{-i \Phi}\right)$ phase factor.

Up to some (unimportant) global phase factor, we have, for the reflection amplitude,

$$
\begin{aligned}
R= & r+t^{2} r\left(e^{2 i \Phi_{u}}+e^{2 i \Phi_{d}}\right) \\
& +t^{3} e^{i\left(\Phi_{u}+\Phi_{d}\right)}\left(e^{i \Phi}+e^{-i \Phi}\right)+O\left((r, t)^{5}\right),
\end{aligned}
$$

which is graphically illustrated in Fig. 2(a). For the transmission we find

$$
\begin{aligned}
T= & t^{2}\left(e^{i\left(\Phi_{u}+\Phi / 2\right)}+e^{i\left(\Phi_{d}-\Phi / 2\right)}\right) \\
& +t^{2} r^{2}\left(e^{3 i \Phi_{u}}+e^{3 i \Phi_{d}}\right) \\
& +2 t^{3} r\left(e^{2 i \Phi_{u}} e^{i\left(\Phi_{d}-\Phi / 2\right)}+e^{2 i \Phi_{d}} e^{i\left(\Phi_{u}+\Phi / 2\right)}\right) \\
& +t^{4}\left(e^{2 i \Phi_{u}} e^{i\left(\Phi_{d}+3 \Phi / 2\right)}\right)+\left(e^{2 i \Phi_{d}} e^{i\left(\Phi_{u}-3 \Phi / 2\right)}\right) \\
& +O\left((r, t)^{6}\right)
\end{aligned}
$$

up to contributions of higher order in powers of $r$ and $t$. Reflection and transmission probabilities are obtained from the amplitudes $R$ and $T$ by calculating their modulus square. They thus involve double sums over trajectories. To observe Al'tshuler-Aronov-Spivak oscillations in these probabilities, a further average over disorder is required. From the original double sum over trajectories, only those pairs of trajectories survive this disorder average that have zero net power of the complex random numbers $e^{i \Phi_{u}}$ and $e^{i \Phi_{d}}$, as can be seen in Fig. 2(b). The disorder-averaged reflection and transmission are then written as

$$
\begin{aligned}
\overline{|R|^{2}}= & |r|^{2}+2|t|^{4}|r|^{2}+2|t|^{6}(1+\cos 2 \Phi)+O\left((r, t)^{10}\right) \\
\overline{|T|^{2}}= & 2|t|^{4}+2|t|^{4}|r|^{4}+8|t|^{6}|r|^{2}+2|t|^{8} \\
& +4|t|^{6}\left(t r^{*}+r t^{*}\right) \cos 2 \Phi+O\left((r, t)^{12}\right)
\end{aligned}
$$

In those expressions, pairs of paths that could give rise to a $\cos (\Phi)$ contribution display a nonzero net power of the 
complex random numbers $e^{i \Phi_{u}}$ and $e^{i \Phi_{d}}$. They do not survive the disorder averaging. Evaluating in the expression (27) the transmission

$$
t r^{*}+r t^{*}=-\frac{\sin ^{2} k \delta}{\left|1-\frac{E_{Y}}{E_{\delta}}+e^{i k \delta}-\frac{1}{2} e^{-i k \delta}\right|^{2}},
$$

we see that $\cos (2 \Phi)$ oscillations of reflection probability are compensated on the transmitted side with nearly the same magnitude (at this level of approximation). We specifically obtain, for $E_{Y}=E_{\delta}$,

$$
2\left(t r^{*}+r t^{*}\right)=-\frac{72}{97} \simeq-0.8 .
$$

This robust enhancement of the reflection, along with the associated drop in the transmission, arises due to coherent backscattering and is a clear signature of weak localization. Similar results have been obtained for quasi-one-dimensional disordered electronic systems in the presence of a magnetic field [28] or in two-dimensional arrays of nonsuperconducting metallic rings [29]. While such oscillations are usually encountered in quasi-one-dimensional models [22-29], we choose here to restrict the motion to exactly one dimension (i.e., we consider a waveguide that does not allow for the population of excited transverse modes at the chemical potential under consideration) since the use of ultracold gases makes this choice possible.

\section{NUMERICAL METHODS}

\section{A. Mean-field Gross-Pitaevskii approach}

The Gross-Pitaevskii approximation has been used for the numerical simulation of atom-laser scenarios [67-71] and it was proven [72] that it is a good approximation in the limit of a large atomic density and small interaction strength. Starting from our Hamiltonian given in Eq. (5) and working in the Heisenberg picture, we obtain the evolution of the annihilation operators according to

$$
\begin{aligned}
i \hbar \frac{\partial \hat{a}_{\alpha}(t)}{\partial t}=\left(E_{\alpha}+V_{\alpha}\right) \hat{a}_{\alpha}(t)-\sum_{\alpha^{\prime}} J_{\alpha \alpha^{\prime}} \hat{a}_{\alpha^{\prime}}(t) & \\
& +g_{\alpha} \hat{a}_{\alpha}^{\dagger}(t) \hat{a}_{\alpha}(t) \hat{a}_{\alpha}(t)+\kappa(t) \delta_{\alpha, \alpha_{\mathcal{S}}} \hat{b}(t) \\
i \hbar \frac{\partial \hat{b}(t)}{\partial t}= & \mu \hat{b}(t)+\kappa^{*}(t) \hat{a}_{\alpha_{\mathcal{S}}}(t)
\end{aligned}
$$

where $J_{\alpha \alpha^{\prime}}$ encodes the matrix elements describing hopping from one site to another within the leads, the ring, and the junction. Additionally, they include the Peierls phase within the ring.

The mean-field limit consists in the regime where the on-site densities are large and the interaction strength is weak. This allows one to replace the quantum operators by $c$-numbers. In that limit, the dynamics of the system is governed by the Gross-Pitaevskii equation

$$
\begin{aligned}
i \hbar \frac{\partial \psi_{\alpha}(t)}{\partial t}= & \left(E_{\alpha}+V_{\alpha}-\mu\right) \psi_{\alpha}(t)-\sum_{\alpha^{\prime}} J_{\alpha \alpha^{\prime}} \psi_{\alpha^{\prime}}(t) \\
& +g_{\alpha}\left|\psi_{\alpha}(t)\right|^{2} \psi_{\alpha}(t)+\kappa(t) \delta_{\alpha, \alpha_{\mathcal{S}}} \chi(t), \\
i \hbar \frac{\partial \chi(t)}{\partial t}= & \kappa^{*}(t) \psi_{\alpha_{\mathcal{S}}}(t),
\end{aligned}
$$

where we have made the ansatz $\psi_{\alpha}(t)=\left\langle\hat{a}_{\alpha}\right\rangle e^{-i \mu t}$ and $\chi(t)=$ $\langle\hat{b}\rangle e^{-i \mu t}$, with $\psi_{\alpha}\left(t_{0}\right)=0$ and $\chi\left(t_{0}\right)=\sqrt{\mathcal{N}}$, corresponding to empty waveguides, an empty ring, and a coherent BoseEinstein condensate within the reservoir of atoms.

It is clear from Eq. (33) that $\chi(t)=\sqrt{\mathcal{N}}\left[1+O\left(|\kappa|^{2}\right)\right]$ for some finite time $t-t_{0}$. Therefore, in the limit where the coupling $\kappa(t)$ tends to zero in such a manner that $\mathcal{N}|\kappa(t)|^{2}$ remains constant, we can neglect the time evolution of $\chi(t)$ and we are left with a nonlinear Schrödinger equation containing an additional source term $[47,69,71]$

$$
\begin{aligned}
i \hbar \frac{\partial \psi_{\alpha}(t)}{\partial t}= & \left(E_{\alpha}+V_{\alpha}-\mu\right) \psi_{\alpha}(t)-\sum_{\alpha^{\prime}} J_{\alpha \alpha^{\prime}} \psi_{\alpha^{\prime}}(t) \\
& +g_{\alpha}\left|\psi_{\alpha}\right|^{2} \psi_{\alpha}(t)+\kappa(t) \delta_{\alpha, \alpha_{\mathcal{S}}} \sqrt{\mathcal{N}}
\end{aligned}
$$

The on-site density and current are defined as

$$
\begin{aligned}
n_{\alpha} & =\left|\psi_{\alpha}\right|^{2}, \\
j_{\alpha} & =\frac{i E_{\delta}}{2 \hbar}\left[\psi_{\alpha+1}^{*}(t) \psi_{\alpha}(t)-\psi_{\alpha}^{*}(t) \psi_{\alpha+1}(t)\right] .
\end{aligned}
$$

The main drawback of this approach is that, notably, when disordered potentials are considered, even a weak atom-atom interaction can generate the population of a noncondensed cloud off the energy shell through two-body scattering [18,63,73]. Those effects are beyond the scope of the mean-field GrossPitaevskii approach and must be addressed by means of another method.

\section{B. Truncated Wigner method}

The truncated Wigner method [74-79], which has been successfully adapted to the context of an atom-laser scenario $[58,80]$, allows one to go beyond the mean-field approximation described by the Gross-Pitaevskii approach. The principle of the method consists in sampling the many-body quantum state of the system by classical fields $\left\{\psi_{\alpha}\right\}_{\alpha \in \mathcal{R}, \mathcal{L}}$ that properly represent the initial state of the system at the initial time $t_{0}$ and evolve according to a slightly modified Gross-Pitaevskii equation. As we consider that initially, at $t=t_{0}$, the waveguides and the ring are empty while the reservoir is populated with a large number $\mathcal{N}$ of atoms, we can decouple the initial Wigner function of the system as

$$
\mathcal{W}\left(\left\{\psi_{\alpha}, \psi_{\alpha}^{*}\right\}, t_{0}\right)=\mathcal{W}_{G}\left(\left\{\psi_{\alpha}, \psi_{\alpha}^{*}\right\}, t_{0}\right) \mathcal{W}_{S}\left(\chi, \chi^{*}, t_{0}\right),
$$

that is, as a product of the Wigner functions describing the source and the scattering system. Initially, the waveguides and the ring are empty, which implies that their Wigner function can be written as a product of vacuum states

$$
\mathcal{W}_{G}\left(\left\{\psi_{\alpha}, \psi_{\alpha}^{*}\right\}, t_{0}\right)=\prod_{\alpha}\left(\frac{2}{\pi}\right) e^{-2\left|\psi_{\alpha}\right|^{2}} .
$$

In practice, the classical field amplitudes are determined as

$$
\psi_{\alpha}\left(t=t_{0}\right)=\frac{1}{2}\left(\mathcal{A}_{\alpha}+i \mathcal{B}_{\alpha}\right)
$$

where $\mathcal{A}_{\alpha}$ and $\mathcal{B}_{\alpha}$ are real and independent Gaussian random variables fulfilling

$$
\begin{aligned}
\overline{\mathcal{A}_{\alpha}} & =\overline{\mathcal{B}_{\alpha}}=0, \\
\overline{\mathcal{A}_{\alpha^{\prime}} \mathcal{A}_{\alpha}} & =\overline{\mathcal{B}_{\alpha^{\prime}} \mathcal{B}_{\alpha}}=\delta_{\alpha^{\prime}, \alpha}, \\
\overline{\mathcal{A}_{\alpha^{\prime}} \mathcal{B}_{\alpha}} & =0
\end{aligned}
$$


in which the overline means that an average over the random variables is performed. Because of that, each site of the system but the source exhibits an artificial nonzero average population $\overline{\left|\psi_{\alpha}\left(t_{0}\right)\right|^{2}}=\frac{1}{2}$ which one has to subtract when computing the atomic density.

As the source is assumed to be populated with a large number $|\chi|^{2}=\mathcal{N} \gg 1$ of atoms, the Wigner function of the source can be considered as that of a coherent state

$$
\mathcal{W}_{\mathcal{S}}\left(\chi, \chi^{*}, t_{0}\right)=\left(\frac{2}{\pi}\right) e^{-2|\chi-\sqrt{\mathcal{N}}|^{2}} .
$$

This very high number of atoms is such that the relative uncertainties on the amplitude and the phase of the source are negligible; we can then treat the source in a classical manner so that $\chi\left(t=t_{0}\right)=\sqrt{\mathcal{N}}$. If in addition the coupling $\kappa(t)$ is chosen such that $\kappa(t) \rightarrow 0$ in such a manner that $\mathcal{N}|\kappa|^{2}$ remains finite, the depletion of the source or any backaction of the waveguide on the source can be safely neglected [58] and one can solely focus on the evolution within the waveguides and the ring. In this case, the propagation equation for the amplitude on each sampling point is given by

$$
\begin{aligned}
i \hbar \frac{\partial \psi_{\alpha}}{\partial t}= & \left(E_{\delta}-\mu+V_{\alpha}\right) \psi_{\alpha}+\sum_{\alpha^{\prime}} J_{\alpha \alpha^{\prime}} \psi_{\alpha^{\prime}} \\
& +g_{\alpha}\left(\left|\psi_{\alpha}\right|^{2}-1\right) \psi_{\alpha}+\kappa(t) \sqrt{\mathcal{N}} \delta_{\alpha, \alpha_{\mathcal{S}}},
\end{aligned}
$$

where $J_{\alpha \alpha^{\prime}}$ are the hopping matrix elements from site $\alpha$ to site $\alpha^{\prime}$.

Observables are computed through an average over the random initial conditions. This, for instance, yields the on-site density and current

$$
\begin{aligned}
n_{\alpha} & =\overline{\left|\psi_{\alpha}\right|^{2}}-\frac{1}{2}, \\
j_{\alpha} & =\frac{i E_{\delta}}{2 \hbar} \overline{\psi_{\alpha+1}^{*}(t) \psi_{\alpha}(t)-\psi_{\alpha}^{*}(t) \psi_{\alpha+1}(t)},
\end{aligned}
$$

where the subtraction of $\frac{1}{2}$ in the density compensates for the artificial $\frac{1}{2}$ atom per site, as explained above.

The truncated Wigner method, in great contrast to a meanfield approach, allows one to access both coherent and incoherent quantities. The coherent contributions to the on-site density and the current are given by

$$
\begin{aligned}
n_{\alpha}^{\mathrm{coh}} & =\left|\overline{\psi_{\alpha}}\right|^{2}, \\
j_{\alpha}^{\mathrm{coh}} & =\frac{i E_{\delta}}{2 \hbar}\left[\overline{\psi_{\alpha+1}^{*}(t)} \overline{\psi_{\alpha}(t)}-\overline{\psi_{\alpha}^{*}(t)} \overline{\psi_{\alpha+1}(t)}\right]
\end{aligned}
$$

and the incoherent ones are then obtained through

$$
\begin{gathered}
n_{\alpha}^{\text {incoh }}=n_{\alpha}-n_{\alpha}^{\text {coh }}, \\
j_{\alpha}^{\text {incoh }}=j_{\alpha}-j_{\alpha}^{\text {coh }} .
\end{gathered}
$$

The transmission is defined as the ratio between the current at a given site in the downstream region behind the ring and the free stationary current

$$
|T|^{2}=\lim _{t \rightarrow \infty} j(t) / j^{\varnothing} .
$$

The reflection is obtained by considering that

$$
|R|^{2}+|T|^{2}=1 \text {. }
$$

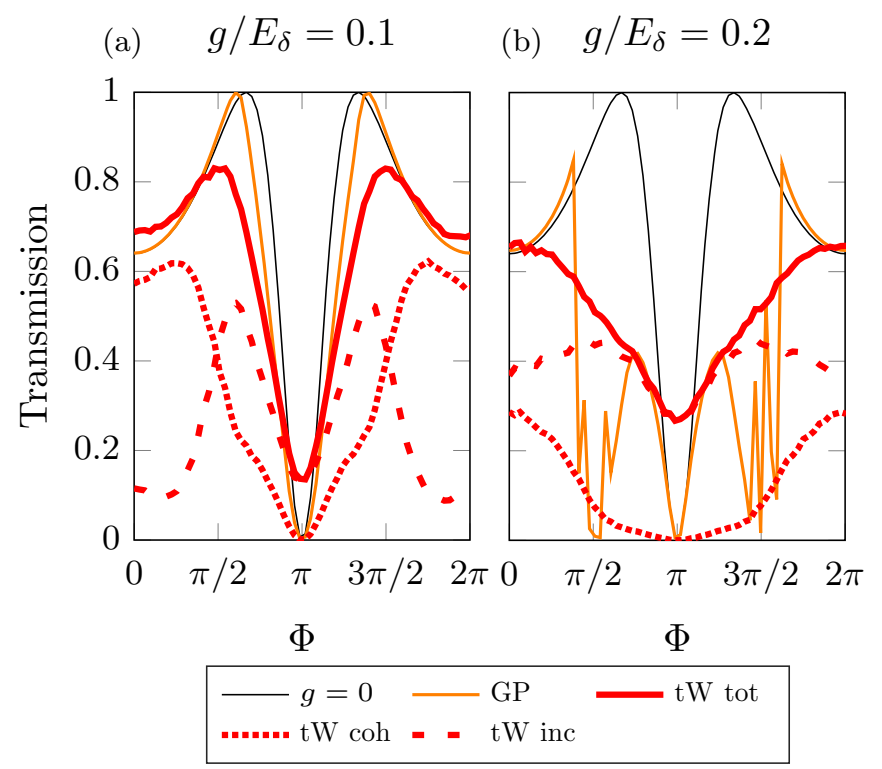

FIG. 3. Noninteracting $\left(g / E_{\delta}=0\right.$, thin black curve), GrossPitaevskii [orange (light gray) curve] and truncated Wigner [red (dark gray) curve] simulations showing the transmission versus $\Phi$ with $\mu / E_{\delta}=1, \sqrt{\mathcal{N}}|\kappa| / E_{\delta}=1$, and (a) $g / E_{\delta}=0.1$ and (b) $g / E_{\delta}=$ 0.2 . A tiny ring with $N_{R}=6$ sites was considered. The noninteracting (black) curve shows the typical Aharonov-Bohm oscillations as a result of interferences at the exit of the ring. Perfect transmission is reached for $\Phi \simeq 3 \pi / 5$ and a transmission blockade is observed at $\Phi=\pi$. (a) While Gross-Pitaevskii simulation essentially confirm this behavior for weak interaction, the truncated Wigner curves show that perfect transmission is inhibited and the transmission blockade at $\Phi=\pi$ is removed, as a result of creation of incoherent particles within the ring. (b) For stronger interactions, we also observe oscillations in the Gross-Pitaevskii transmission [orange (light gray) curve] due to bistability that indicates a breakdown of matter wave coherence. This is confirmed by truncated Wigner simulations yielding dominantly incoherent contributions.

Similarly as for the density and the current, one can also introduce the coherent and incoherent part of the transmission as

$$
\begin{aligned}
T^{\mathrm{coh}} & =\lim _{t \rightarrow \infty} j(t)^{\mathrm{coh}} / j^{\varnothing}, \\
T^{\mathrm{incoh}} & =\lim _{t \rightarrow \infty} j(t)^{\mathrm{incoh}} / j^{\varnothing} .
\end{aligned}
$$

\section{RESULTS}

\section{A. Aharonov-Bohm oscillations}

We begin our numerical study by setting the disorder strength to zero, which allows us to focus solely on the interplay of interference and interaction. This is the standard Aharonov-Bohm scenario where the interference between the semiclassical contributions resulting for the two arms can be constructive or destructive depending on the artificial flux $\Phi$ within the ring. This can be verified by computing the transmission, which is defined by the ratio between the current at a site located after the ring and the injected free current $j^{\varnothing}$ defined in Eq. (7).

The expected interference pattern is confirmed in Fig. 3. The noninteracting (black) curve shows the steady-state result 
of a simulation of the transmission as a function of the artificial flux $\Phi$ for $g=0$. For $\Phi \simeq 3 \pi / 5$, the transmission of atoms is perfect and reaches one, which is a signature of destructive interference of the reflection at the entry side. On the other hand, the transmission blockade at $\Phi=\pi$ highlights destructive interferences at the exit site of the ring, namely, between the partial waves crossing each arm of the ring, giving rise to a transmission blockade. Performing a GrossPitaevskii simulation for a small interaction $\left[g / E_{\delta}=0.1\right.$, orange (light gray) curve], we observe a displacement of the maxima but no lifting of the transmission blockade. Truncated Wigner simulations reveal that a suspension of the blockade occurs at $\Phi=\pi$. This is entirely due to the incoherent part of the transmission, which could not have been predicted by Gross-Pitaevskii simulations. Interaction is responsible for this incoherent transmission because noncondensed particles, with kinetic energy slightly lower or higher than that of the condensate, are created within the ring as a result of interaction.

Figure 3(b) shows the transmission for larger g. Oscillations in the Gross-Pitaevskii curve can be seen as an artifact of the mean-field approach. Those oscillations are a signature of bistability, as documented and observed in [58,69,71]. Truncated Wigner simulations reveal a breakdown of matter wave coherence. Aharonov-Bohm-like oscillations are nevertheless encountered owing to significant remnants of coherent components of the atomic cloud near $\Phi=0$ and $\Phi=2 \pi$. For stronger interactions, Aharonov-Bohm oscillations are expected to be washed out according to the study undertaken in Ref. [81].

\section{B. From Aharonov-Bohm to Al'tshuler-Aronov-Spivak oscillations}

As explained in Sec. III, if we add a smooth (for instance, Gaussian-correlated) disorder potential within the ring, we can cancel the Aharonov-Bohm oscillations in the transmission and reveal Al'tshuler-Aronov-Spivak ones [22-25]. Indeed, a random phase is acquired after a trip in the ring due to the fact that the disorder potentials in the upper and lower arms of the ring are not correlated with each other. For pairings of trajectories that provide contributions to AharonovBohm oscillations, the phase averages out and such pairings do not contribute on average. On the other hand, as pairings of trajectories that provide contributions to Al'tshuler-AronovSpivak oscillations, which are time-reversed conjugates of each other, are such that the same phase is accumulated, such pairings do not cancel with each other and are preserved after averaging.

In order to develop a nonlinear diagrammatic theory taking into account interaction effects, we want to work in the semiclassical regime which corresponds to an action $S \gg \hbar$ and a correlation length $\sigma \gg \lambda$. For that purpose, we need to simultaneously enforce the four conditions

$$
\delta \ll \lambda \ll \sigma \ll L \ll l_{\mathrm{loc}},
$$

where $l_{\text {loc }} \propto \exp \left(4 k^{2} \sigma^{2}\right)$ [62] is the localization length for strong (Anderson) localization [82] within an arm of the ring and $L$ is the length of the ring. Furthermore, $\mu$ has to be small compared to $E_{\delta}$ to be close to the free dispersion relation of the

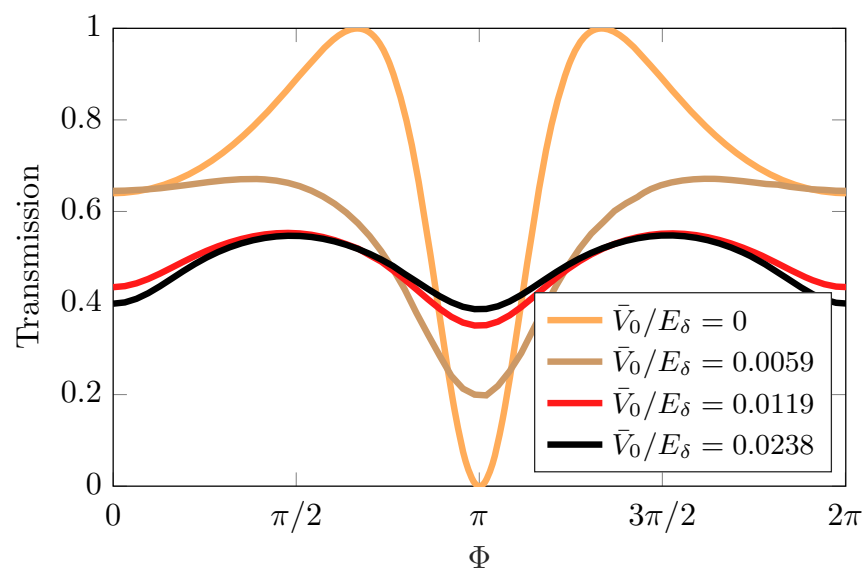

FIG. 4. Noninteracting simulation showing the transmission versus $\Phi$ with $\mu / E_{\delta}=0.2$ and $N_{R}=200$ sites. We considered 20000 realizations of a Gaussian-correlated disorder according to Eq. (9) with the amplitude $\bar{V}_{0}$ and correlation length $\sigma=20 \delta$. AharonovBohm oscillations [orange (light gray) curve] of period $2 \pi$ smoothly turn into Al'tshuler-Aronov-Spivak oscillations [black (dark gray) curve] of period $\pi$ as the disorder strength is increased.

continuous one-dimensional space. Specifically, we choose the chemical potential $\mu / E_{\delta}=0.2$, the disorder amplitude $\bar{V}_{0}=0.0238$, and the correlation length $\sigma=20 \delta$. We indeed have $k \delta \approx 0.67<1$ and $k \sigma \approx 13.4 \gg 1$, indicating that we are working in the validity regime of semiclassical methods.

As is shown in Fig. 4, Aharonov-Bohm oscillations are washed out by the ensemble average, giving rise to Al'tshulerAronov-Spivak oscillations of period $\pi$. Figure 5 shows the disorder-averaged density of atoms on each site. It illustrates robust interferences that take place near the entrance and exit junctions of the ring. They arise because reflected particles interfere with the injected current. Deep inside the ring as well as in the downstream region, on the other hand, a homogeneous mean density is encountered.
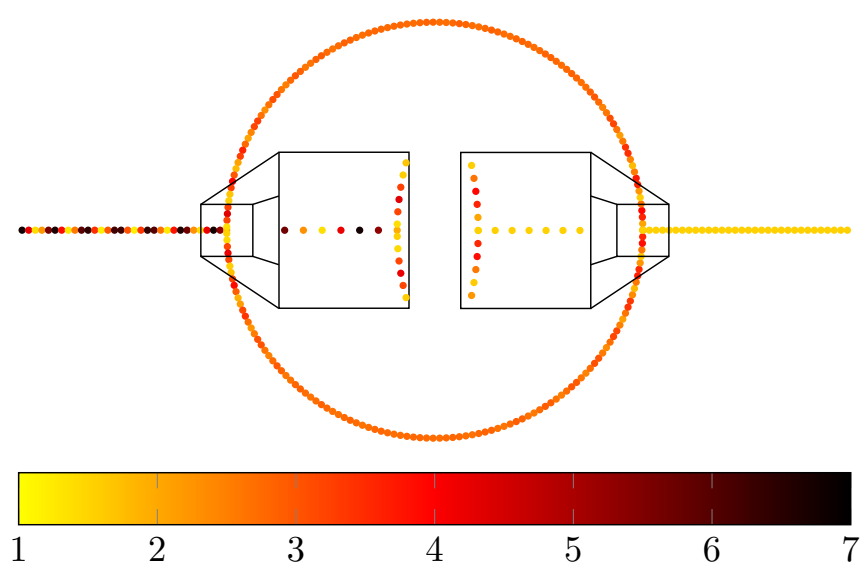

FIG. 5. Disorder averaged density of atoms at each site of the system at $\Phi=\pi / 2$ in a noninteracting situation with $\mu / E_{\delta}=0.2$ and $N_{R}=200$ sites. We considered 20000 realizations of a Gaussian-correlated disorder taking random values in $[-0.0119,0.0119]$ with correlation length $\sigma=20 \delta$. Systematic interferences between particles take place at the entrance and exit junctions of the ring. 


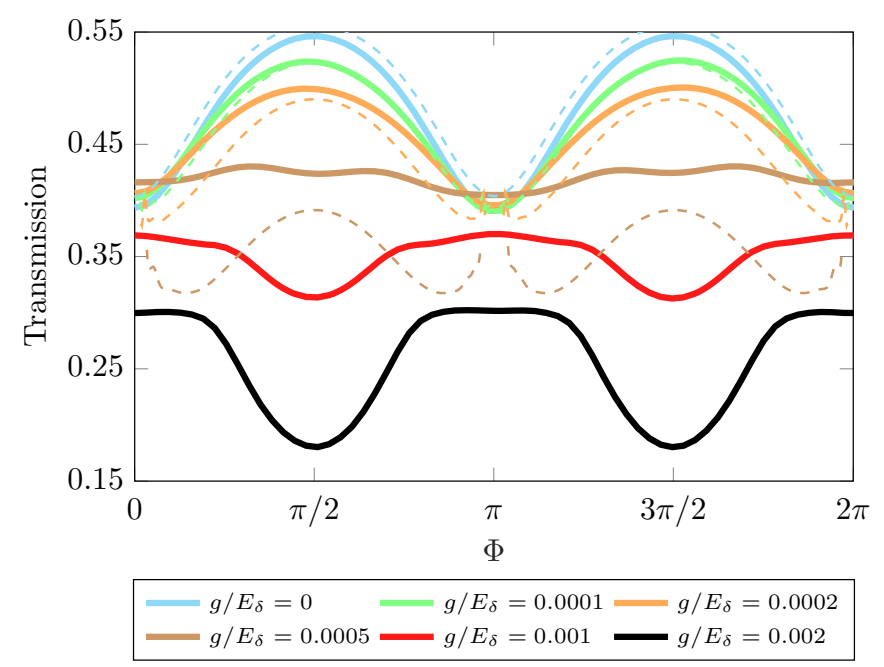

FIG. 6. Gross-Pitaevskii simulations showing the transmission versus $\Phi$ for different interaction strengths $g$ (increasing $g$ with increasing grayscale) with $\mu / E_{\delta}=0.2, N_{R}=200$ sites, and $\sqrt{\mathcal{N}}|\kappa| / E_{\delta}=1$, which yields a density $\delta \rho^{\varnothing}=2.77$. We considered 20000 realizations of a Gaussian-correlated disorder taking random values in $[-0.0119,0.0119]$ with correlation length $\sigma=20 \delta$. The interaction gives first rise to a flattening and then to a reversal of the curve: The maxima at $\Phi=\pi / 2$ and $3 \pi / 2$ become minima and the minima at $\Phi=0$ and $\pi$ become maxima. The predictions of our diagrammatic theory are represented in dashed lines of the same color as the corresponding Gross-Pitaevskii simulations. They exhibit good agreement with the latter for low values of the interaction strength. As soon as $g$ increases, quadratic corrections become more important and the predictions of a linear theory become less reliable.

\section{Competition between disorder and interaction effects}

Figure 6 shows the results of a Gross-Pitaevskii simulation for different values of the interaction strength $g$ showing the transmission as a function of $\Phi$. We see that from $g / E_{\delta}=0$ to $g / E_{\delta}=0.0005$, the presence of interaction gives rise to a flattening of the oscillations by reducing the amplitude. However, for $g / E_{\delta}=0.001$ and $g / E_{\delta}=0.002$, we observe an inversion of Al'tshuler-Aronov-Spivak oscillations. This is in qualitative agreement with coherent backscattering inversion [15]. The minima of transmission at $\Phi=0$ and $\pi$ (corresponding to maxima of reflection in a coherent backscattering scenario) become maxima and the two maxima located around $\Phi=\pi / 2$ and $3 \pi / 2$ become minima. We expect that those results remain quantitatively the same in a large range of parameters defining the disorder potential, provided the latter is sufficiently strong to fully randomize the phase factors $e^{i \Phi_{u}}$ and $e^{i \Phi_{d}}$ and at the same time sufficiently weak and smooth so that it does not induce (partial or total) reflections within each arm of the ring.

We also plotted in this figure the predictions of our firstorder-in- $g$ diagrammatic theory which will be developed in Sec. VI. The agreement we find between the two curves is reasonably good for weak interaction strength $g / E_{\delta} \lesssim 0.0002$. At stronger interactions, significant deviations occur due to quadratic corrections becoming more important.

Another comparison with the predictions of our analytical diagrammatic theory is shown in Fig. 7, where we plot the

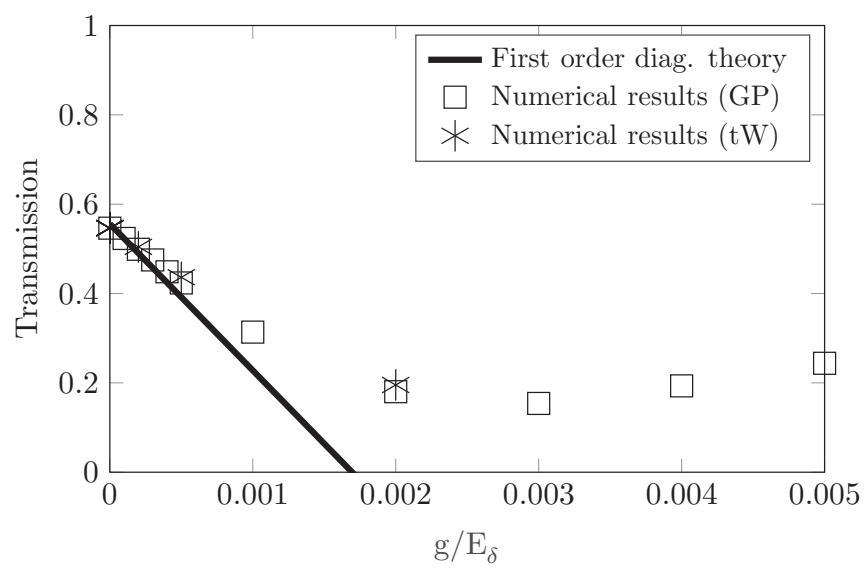

FIG. 7. Comparison between the first-order diagrammatic theory predictions and the results of numerical Gross-Pitaevskii and truncated Wigner simulations for the transmission as a function of the interaction strength $g$ at $\Phi=\pi / 2$, with $\mu / E_{\delta}=0.2$ and $N_{R}=200$ sites. We considered 20000 realizations of a Gaussian-correlated disorder taking random values in $[-0.0119,0.0119]$ with correlation length $\sigma=20 \delta$. Good agreement is found for weak interaction strength where the transmission decreases approximately linearly with $g$.

transmission at $\Phi=\pi / 2$ at a finite value of the interaction strength. We find that for small values of $g$, the transmission decreases linearly with $g$. The initial decrease of the transmission with $g$ is reasonably well predicted by our diagrammatic theory at about $g \simeq 10^{-3} E_{\delta}$, beyond which quadratic corrections in $g$ become important. A maximal inversion of Al'tshuler-Aronov-Spivak oscillations is reached at about $\Phi \simeq 0.003 E_{\delta}$.

Finally, we perform truncated Wigner simulations in a regime where the inversion of Al'tshuler-Aronov-Spivak oscillations is fully developed, namely, for $g / E_{\delta}=0.002$. We therefore perform six sets of simulations, with different values for both the density $\rho^{\varnothing}$ and $g$, their product being kept constant and equal to $\delta g \rho^{\varnothing} / E_{\delta} \simeq 0.0055$. The results of these simulations are shown in Fig. 8. We clearly see that truncated Wigner simulations predict a flattening of the inverted Al'tshuler-Aronov-Spivak transmission profile, corresponding to a complete dephasing of quantum interference effects.

We furthermore see that the inverted Al'tshuler-AronovSpivak oscillation structure arises entirely due to a coherent contribution, thereby confirming that the inversion of the central minimum has the same origin as coherent backscattering inversion. The coherent part formally still exhibits this inverted structure, but is hidden behind the incoherent contribution which has become very large, indicating the presence of dephasing for strong interaction.

\section{DIAGRAMMATIC THEORY FOR THE COHERENT CONTRIBUTIONS TO THE AL'TSHULER-ARONOV-SPIVAK OSCILLATIONS}

\section{A. Formal solution of the Gross-Pitaevskii equation}

The starting point of an analytical diagrammatic theory on the mean-field level is the Gross-Pitaevskii equation of the 


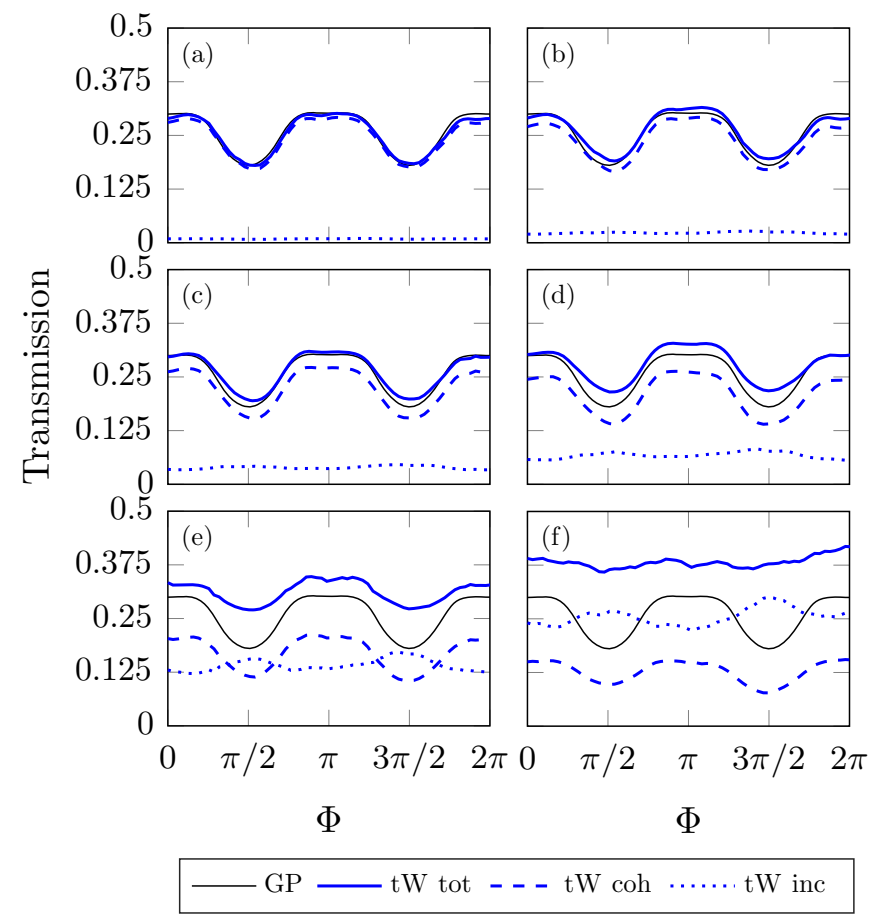

FIG. 8. Truncated Wigner simulation showing the transmission versus $\Phi$ for different interaction strengths $g$ and $\rho^{\varnothing}$, the product $\delta g \rho^{\varnothing} / E_{\delta} \simeq 0.0055$ being kept constant all the way. In the GrossPitaevskii simulations (thin black line), this corresponds to a regime where the inversion of Al'tshuler-Spivak oscillations is fully developed. The simulation parameters are $\mu / E_{\delta}=0.2$ and $N_{R}=200$ sites. We considered 20000 realizations of a Gaussian-correlated disorder taking random values in $[-0.0119,0.0119]$ with correlation length $\sigma=20 \delta$, each of which is done with an average of 100 realizations over the initial conditions. The values of $g / E_{\delta}$ and $\delta \rho^{\varnothing}$ are (a) $g / E_{\delta}=0.0002$ and $\delta \rho^{\varnothing} \simeq 27.77$, (b) $g / E_{\delta}=0.001$ and $\delta \rho^{\varnothing} \simeq 5.55$, (c) $g / E_{\delta}=0.002$ and $\delta \rho^{\varnothing} \simeq 2.77$, (d) $g / E_{\delta}=0.004$ and $\delta \rho^{\varnothing} \simeq 1.38$, (e) $g / E_{\delta}=0.01$ and $\delta \rho^{\varnothing} \simeq 0.55$, and (f) $g / E_{\delta}=$ 0.02 and $\delta \rho^{\varnothing} \simeq 0.27$.

discretized system, which is given by Eq. (34). This equation is formally solved by the time-dependent scattering wave function

$$
\psi_{\alpha}(t)=\sum_{\alpha^{\prime}} G\left(\alpha, \alpha^{\prime}, \mu\right) S_{\alpha^{\prime}}(t),
$$

where we used the linear Green's function for the system without interaction. The source term and the nonlinear interaction term are contained in

$$
S_{\alpha}(t)=g_{\alpha}\left|\psi_{\alpha}(t)\right|^{2} \psi_{\alpha}(t)+\kappa(t) \delta_{\alpha, \alpha_{\mathcal{S}}} \sqrt{\mathcal{N}}
$$

In the limit of long times, when we have reached a stationary state, the Gross-Pitaevskii equation transforms into a selfconsistent equation

$$
\begin{aligned}
\psi_{\alpha}= & \sqrt{\mathcal{N}} \kappa(t) G\left(\alpha, \alpha_{S}, \mu\right) \\
& +\sum_{\alpha^{\prime}} G\left(\alpha, \alpha^{\prime}, \mu\right) g_{\alpha^{\prime}}\left|\psi_{\alpha^{\prime}}\right|^{2} \psi_{\alpha^{\prime}},
\end{aligned}
$$

which marks the starting point for a perturbation theory in the small interaction parameter $g$. The zeroth order in this expansion marks the noninteracting case.

\section{B. Noninteracting case}

In this section we present the calculation of the Green's function for the noninteracting case and for a fixed disorder configuration. This calculation is based on its representation by a sum over all paths linking two sites (see Appendix A),

$$
G\left(\alpha, \alpha^{\prime}, \mu\right)=\frac{1}{i E_{\delta} \sin (k \delta)} \sum_{\gamma} A_{\gamma} e^{i S_{\gamma} / \hbar} .
$$

To visualize this, it is useful to think of the system as a quantum graph consisting of two semi-infinite waveguides, which are on opposite sides attached to the ring structure via two junctions. Depending on the locations $\alpha$ and $\alpha^{\prime}$ at the beginning and the end of $\gamma$, the path might visit one or both of the junctions and fully explore the branches of the ring multiple times. Every path $\gamma$ in the coherent sum in Eq. (59) may contain an arbitrarily long sequence of such alternating visits of junctions and explorations of branches.

The phase factor $\exp \left(i / \hbar S_{\gamma}\right)$ contains the accumulated phase of the repeated exploration of the branches. A single traversal of a branch contributes a random but fixed phase $\Phi_{u}$ or $\Phi_{d}$ due to the disorder potential. Additionally, we get another phase contribution $\pm \Phi / 2$, whose magnitude depends on the flux enclosed by the ring, and its sign encodes whether the flux is encircled in the counterclockwise or clockwise direction. The crossing of a junction is treated within a scattering approach and yields a multiplicative contribution to the amplitude $A_{\gamma}$, either a reflection $r$ or a transmission amplitude $t$, depending on the geometry of the path $\gamma$ before and after the junction.

To keep track of the contributions of a single path to the coherent sum in Eq. (59), we establish the following diagrammatical representations:

$$
\begin{aligned}
& \boldsymbol{\gamma}=r, \\
& =e^{i\left(\Phi_{\mathrm{u}}+\frac{\Phi}{2}\right)}, \\
& >=e^{i\left(\Phi_{\mathrm{d}}-\frac{\Phi}{2}\right)},
\end{aligned}
$$

Every path can be visualized as a sequence of the above building blocks, where the sequence alternates between the scattering process at a junction [first line in Eq. (60)] and the exploration of one of the branches, in either the clockwise or counterclockwise direction [second and third lines in Eq. (60)].

Our aim is to perform the coherent sum over all paths inherent in the noninteracting Green's function. To do so, we first focus on the sequential part oscillating between the two junctions. We group these sequences depending on which junction they start and end and how they approach and leave the limiting junctions before and after the sequence. This group of sequences with identical limiting conditions is then resummed and represented by a single new diagram in the color orange (dark gray) and an arrow representing the common initial and final behaviors. For instance, the subsequent summation

$$
\begin{aligned}
& \hat{\phi}=\hat{\phi} \vec{\gamma}+\vec{\phi} \\
& +r^{\longrightarrow},+\cdots,
\end{aligned}
$$


represents the sum of all trajectories that approach the left junction from the lower branch, have an arbitrary number of explorations of upper and lower branches of the AharonovBohm ring, and then finally leave to the upper branch. The boundary conditions, as well as the junction they refer to, are encoded in the direction and the curvature of the orange (dark gray) arrow. This way of thinking defines a whole set of resummed diagrams

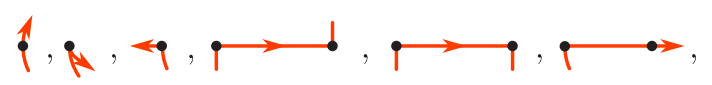

as well as rotated and mirrored versions of the above diagrams. The selected resummed diagrams in Eq. (62) all represent resummations of paths that approach the left junction from the lower branch at the beginning. The first three end at the left junction, while the last three end at the right one. From there, the diagrams either leave to the upper or the lower branch of the ring or exit the ring to the attached waveguide.

The calculation of these resummed diagrams utilizes selfconsistent equations. Those are constructed by the observation that every path contained in the sums of the diagrams (62) can be extended to a longer path by successively adding two branches and two junctions. By appropriately adjusting the boundary conditions, these longer paths have to be an element of a resummed diagram. For instance, we find

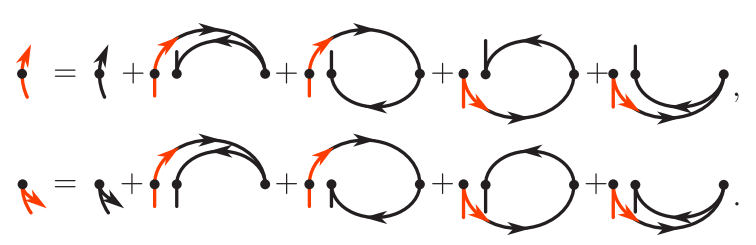

We can rewrite these equations in a diagrammatic matrixvector version

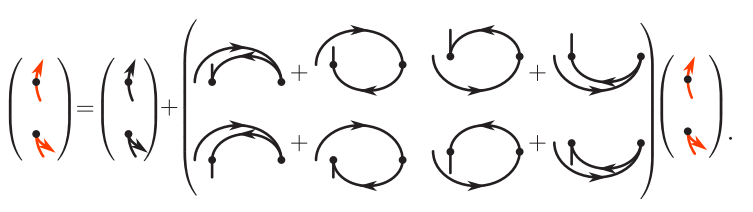

To avoid a repeated use of the same diagrams in the subsequent calculations, let us denote the two-dimensional vector with the resummed diagrams, which appears on both sides of Eq. (64), by $\vec{w}$ and the first vector on the right-hand side of Eq. (64) by $\vec{v}$. The matrix will be denoted by $A$. Then, by applying the rules in Eq. (60) the diagrams translate to $\vec{v}=(t, r)^{\top}$ and

$$
A=\left(\begin{array}{ll}
r^{2} e^{2 i \Phi_{u}}+t^{2} e^{i\left(\Phi_{u}+\Phi_{d}+\Phi\right)} & r t e^{i\left(\Phi_{u}+\Phi_{d}-\Phi\right)}+r t e^{2 i \Phi_{d}} \\
r t e^{2 i \Phi_{u}}+r t e^{i\left(\Phi_{u}+\Phi_{d}+\Phi\right)} & t^{2} e^{i\left(\Phi_{u}+\Phi_{d}-\Phi\right)}+r^{2} e^{2 i \Phi_{d}}
\end{array}\right) .
$$

The self-consistent equation (64) translates to $\vec{w}=\vec{v}+A \vec{w}$, whose solution $\vec{w}$ is found by a simple matrix inversion $\vec{w}=(1-A)^{-1} \vec{v}$. We obtain

$$
\left(\begin{array}{l}
\hat{i} \\
\hat{A}
\end{array}\right)=\frac{1}{D\left(\Phi_{\mathrm{u}}, \Phi_{\mathrm{d}}, \Phi\right)}\left(\begin{array}{c}
t\left(1+\left(r^{2}-t^{2}\right) e^{i\left(\Phi_{\mathrm{u}}+\Phi_{\mathrm{d}}-\Phi\right)}\right) \\
r\left(1-\left(r^{2}-t^{2}\right) e^{2 i \Phi_{\mathrm{u}}}\right)
\end{array}\right),
$$

where the common denominator is the determinant of the matrix $(1-A)$,

$$
\begin{aligned}
D\left(\Phi_{u}, \Phi_{d}, \Phi\right)= & \operatorname{det}(1-A) \\
= & 1-r^{2}\left(e^{2 i \Phi_{u}}+e^{2 i \Phi_{d}}\right)-2 t^{2} e^{i\left(\Phi_{u}+\Phi_{d}\right)} \cos (\Phi) \\
& +\left(r^{2}-t^{2}\right)^{2} e^{2 i\left(\Phi_{u}+\Phi_{d}\right)} .
\end{aligned}
$$

In principle, the other diagrams in Eq. (62) can be calculated in a similar manner, but this is not necessary. Having one pair of resummed diagrams, it is possible to derive all the other diagrams in Eq. (62) as well as their mirrored and rotated versions. To construct a new path from a given path with a different behavior at the initial or final junction, it is sufficient to elongate the original path by one or two branches, thereby incorporating the desired behavior at the new endings. This leads to a set of diagrammatic identities, which we present in Appendix B.

We are now ready to write a diagrammatic expression for the linear Green's function $G\left(\alpha, \alpha^{\prime}, E\right)$. For instance, if the sites $\alpha$ and $\alpha^{\prime}$ are in the upper branch of the ring, we have

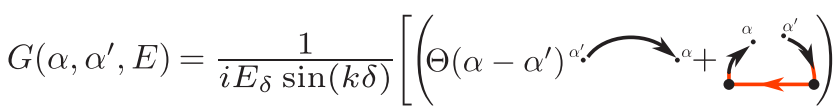

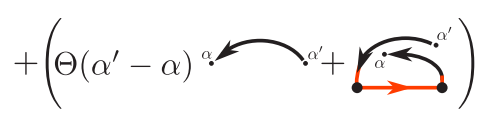

$$
\begin{aligned}
& \left.+\alpha \cdot \frac{\alpha^{\prime}}{\gamma^{\prime}}+\dot{\alpha}^{\prime}\right]
\end{aligned}
$$

where the Heaviside function $\Theta(\cdot)$ distinguishes the cases where $\alpha$ is to the left $\left(\alpha<\alpha^{\prime}\right)$ or right of $\alpha^{\prime}\left(\alpha>\alpha^{\prime}\right)$. Note that as these representations include the partial exploration of a branch, details of the disorder in the single branches are needed to obtain the correct phase. However, as we are interested in transport through the ring, we will see that it is sufficient to consider only paths that terminate at junctions, i.e., which only contain full explorations of the branches of the ring.

For the calculation of the full noninteracting reflection amplitudes $R^{(0)}$ we need to consider a Green's function that starts and ends at a site in the left waveguide. This requires us to sum over all paths that start and end in that waveguide. Utilizing the resummed diagrams, we obtain, up to a constant phase,

$$
\begin{aligned}
R^{(0)}= & =P \cdot+ \\
=r & +\frac{t^{2}}{D\left(\Phi_{\mathrm{u}}, \Phi_{\mathrm{d}}, \Phi\right)}\left[-2(r-t)\left(r^{2}-t^{2}\right) e^{i 2\left(\Phi_{\mathrm{u}}+\Phi_{\mathrm{d}}\right)}\right. \\
& \left.+r\left(e^{i 2 \Phi_{\mathrm{u}}}+e^{i 2 \Phi_{\mathrm{d}}}\right)+2 t e^{i\left(\Phi_{\mathrm{u}}+\Phi_{\mathrm{d}}\right)} \cos (\Phi)\right]
\end{aligned}
$$


In a similar way, we can also calculate the full noninteracting transmission amplitude $T^{(0)}$,

$$
\begin{aligned}
T^{(0)}= & \cdots= \\
= & \frac{t^{2}}{D\left(\Phi_{\mathrm{u}}, \Phi_{\mathrm{d}}, \Phi\right)}\left[e^{i\left(\Phi_{\mathrm{u}}+\frac{\Phi}{2}\right)}+e^{i\left(\Phi_{\mathrm{d}}-\frac{\Phi}{2}\right)}\right. \\
& \left.-(r-t)^{2} e^{i\left(\Phi_{\mathrm{u}}+\Phi_{\mathrm{d}}\right)}\left(e^{i\left(\Phi_{\mathrm{u}}-\frac{\Phi}{2}\right)}+e^{i\left(\Phi_{\mathrm{d}}+\frac{\Phi}{2}\right)}\right)\right] .
\end{aligned}
$$

To obtain from these results the disorder-averaged noninteracting reflection and transmission probability, we have to take the modulus square of the amplitudes in Eqs. (69) and (70) and average them over all disorder phases $\Phi_{u}, \Phi_{d} \in[0,2 \pi]$,

$$
\begin{aligned}
& \overline{\left|R^{(0)}\right|^{2}}(\Phi)=\frac{1}{(2 \pi)^{2}} \int_{0}^{2 \pi} d \Phi_{u} \int_{0}^{2 \pi} d \Phi_{d}\left|R^{0}\left(\Phi_{u}, \Phi_{d}, \Phi\right)\right|^{2} \\
& \overline{\left|T^{(0)}\right|^{2}}(\Phi)=\frac{1}{(2 \pi)^{2}} \int_{0}^{2 \pi} d \Phi_{u} \int_{0}^{2 \pi} d \Phi_{d}\left|T^{(0)}\left(\Phi_{u}, \Phi_{d}, \Phi\right)\right|^{2} .
\end{aligned}
$$

We performed the calculation of those integrals numerically with a Monte Carlo approach. The result of this calculation agrees very well with the numerical simulation, as shown in Fig. 6.

\section{Interacting case}

For the diagrammatic representation of the full interacting solution $\psi_{\alpha}$ [Eq. (58)], we introduce a diagrammatic theory similar to that for the noninteracting case. Our aim is thus to distinguish sets of so to speak interacting trajectories according to whether their final point $\alpha$ in the index of $\psi_{\alpha}$ is in the upper or the lower branch of the Aharonov-Bohm ring and whether $\alpha$ is approached from its left or right side. The wave function $\psi_{\alpha}$ is then written as sum of these diagrams, e.g., for $\alpha$ in the upper part of the ring,

$$
\psi_{\alpha}=\frac{\sqrt{N} \kappa(t)}{i E_{\delta} \sin (k \delta)}\left(\int^{\alpha}+{ }^{\alpha} \cdot\right),
$$

where the green (light gray) diagrams have to be understood in view of the self-consistent equation (58). On the right-hand side of that equation, the final site $\alpha$ is approached by paths inherent in the linear Green's function $G\left(\alpha, \alpha^{\prime}, E\right)$. The two green (light gray) diagrams in Eq. (73) account for those two possibilities, namely, that the final site is approached in the clockwise or counterclockwise direction.

In the Gross-Pitaevskii equation (58), in close analogy to the nonlinear diagrammatic theory for 2D billiards [17], we represent by a box the intermediate site $\alpha^{\prime}$ where the nonlinear wave interacts with itself through the density $\left|\Psi_{\alpha^{\prime}}\right|^{2}$. Complex conjugation is diagrammatically expressed by replacing solid lines with dashed ones. Then Eq. (58) transforms into a set of diagrammatic equations. One of them is

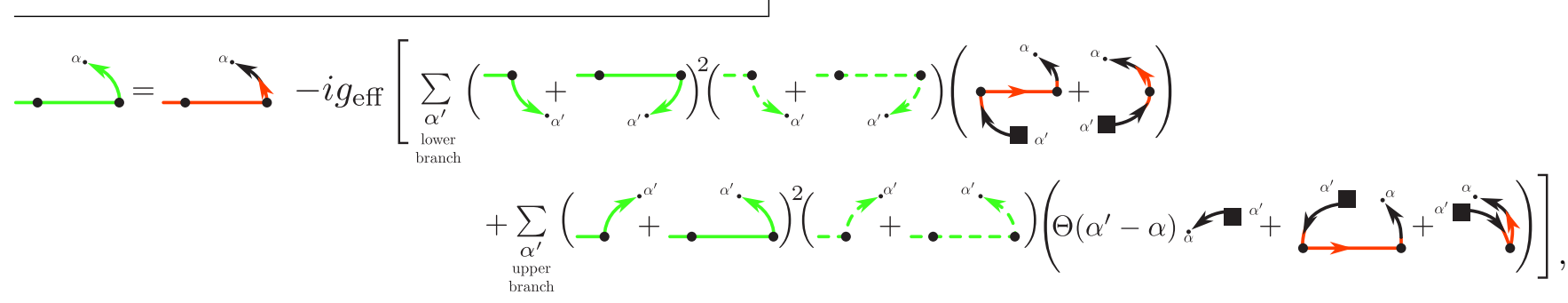

where

$$
g_{\text {eff }}=\frac{g \mathcal{N}|\kappa(t)|^{2}}{\left[E_{\delta} \sin (k \delta)\right]^{3}}
$$

denotes the effective interaction strength. By subsequently inserting the left-hand side into the right-hand side, Eq. (74) and its relatives can be used for a perturbative calculation in the small effective interaction strength $g_{\text {eff }}$.

To obtain the diagrams for the full reflection (transmission) amplitude for the interacting case, we have to send the site $\alpha$ in Eq. (73) to the left (right) junction site and add a transmission event to leave the ring. We obtain

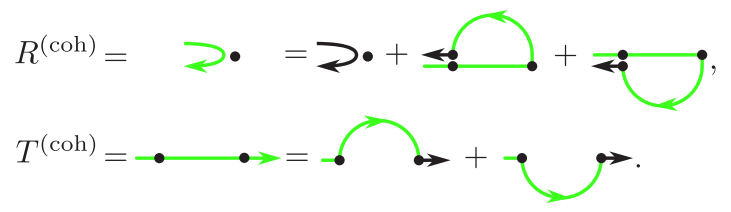

The exact calculation of these expressions in first and higher orders of the effective interaction strength $g_{\text {eff }}$, however, requires details of the specific disorder at the single sites: To perform the summation over all sites in the ring in Eqs. (58) and (74) we have to find the accumulated phase from a partial exploration of one of the branches. However, to arrive at 
the desired averaged reflection and transmission probability, the modulus square of Eq. (76) has to be averaged over disorder configurations. The only contributions surviving the averaging process are those where the accumulated phase of a partial exploration is, with the help of other paths, either extended to the phase of a full exploration or compensated by other partial explorations with an opposite sign in front of their phases. Consequently, this is only the case for path constellations where the nonlinearity event displays one of the structures

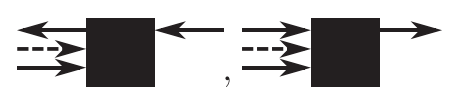

as well as the mirrored and/or complex conjugated versions of those. Contrarily, structures involving

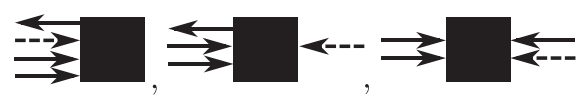

produce an uncompensated accumulated phase which does not survive the disorder average. ${ }^{2}$

The above arguments motivate excluding the structures in Eq. (78) from our diagrammatic theory. This allows us to perform calculations without the need to know exact details of the specific disorder configuration while still producing accurate results for the disorder-averaged reflection and transmission probabilities. Equation (74) is replaced by

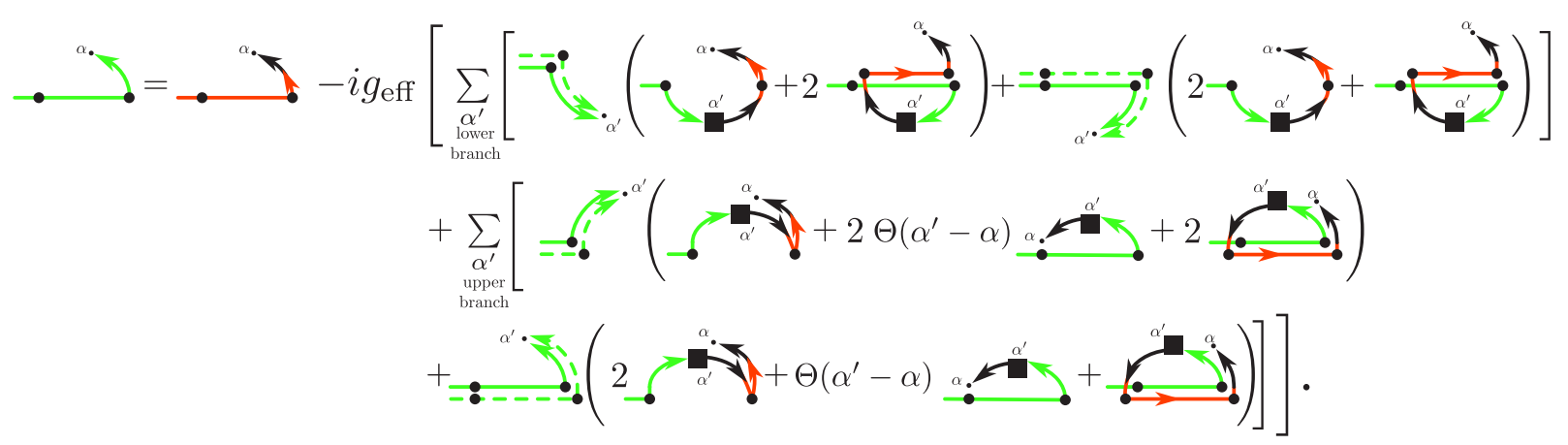

The additional factor 2 appearing in the above terms is a combinatorical factor, reflecting the two possibilities to build the corresponding contributions from the single diagrammatic parts in Eq. (74).

The first order of the perturbative expansion of Eq. (79) in orders of $g_{\text {eff }}$ is obtained by replacing green (light gray) diagrams by orange (dark gray) ones with a subsequent partial exploration of a branch,

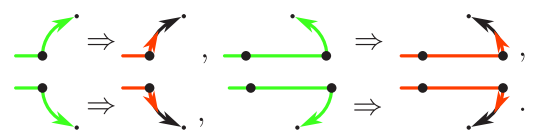

Within this approximation, the terms in the summation over the intermediate site $\alpha^{\prime}$ in Eq. (79) are independent of $\alpha^{\prime}$ and the summation is easily performed, yielding an additional factor $N_{R}$, the number of sites in a single branch of the ring.

By further utilizing diagrammatic equalities for resummed paths, we obtain that the effective corrections in first order in $g_{\text {eff }}$ to the reflection and transmission amplitudes are diagrammatically given by

$$
\delta \tilde{R}^{(1)}=-i g_{\mathrm{eff}} \frac{N_{R}}{2}[\text { ( }
$$

\footnotetext{
${ }^{2}$ In principle, to compensate for the phase of one of the forbidden structures, a second nonlinearity event, which moves together with the first, would be needed. However, this requires one to reduce two summations over the positions of the nonlinearity events to a single one. Their contribution is thus suppressed by a factor of the order of the inverse number of sites compared to those including only allowed structures.
} 
By using the calculated analytical expressions for the appearing resummed diagrams, we can obtain analytical expressions for these contributions. These expressions can then be used to numerically calculate the first-order correction according to

$$
\begin{aligned}
& \delta \overline{\left|R^{(1)}\right|^{2}}(\Phi)=\overline{\left|R^{(1)}\right|^{2}}(\Phi)-\overline{\left|R^{(0)}\right|^{2}}(\Phi) \\
&=\iint_{0}^{2 \pi} \frac{d \Phi_{u} d \Phi_{d}}{(2 \pi)^{2}} 2 \operatorname{Re}\left[R^{(0) *}\left(\Phi_{u}, \Phi_{d}, \Phi\right)\right. \\
& \delta \overline{\left|T^{(1)}\right|^{2}}(\Phi)=\overline{\left|T^{(1)}\right|^{2}}(\Phi)-\overline{\left|R^{(0)}\right|^{2}}(\Phi) \\
&=\iint_{0}^{2 \pi} \frac{d \Phi_{u} d \Phi_{d}}{(2 \pi)^{2}} 2 \operatorname{Re}\left[T^{(0) *}\left(\Phi_{u}, \Phi_{d}, \Phi\right)\right. \\
&\left.\delta \tilde{T}^{(1)}\left(\Phi_{u}, \Phi_{d}, \Phi\right)\right] .
\end{aligned}
$$

Like for the noninteracting case, we carried out the above averaging through a numerical integration using a Monte Carlo method. Nonzero first-order corrections are obtained essentially owing to the complexity of the reflection and transmission matrix elements at a $Y$ junction, according to Eqs. (17) and (18). Note that they would vanish for the choice of a widely open ring with $r=-\frac{1}{3}$ and $t=\frac{2}{3}$ [Eqs. (15) and (16)].

\section{CONCLUSION}

In summary, we numerically studied the 1D transport of a Bose-Einstein condensate through a lead connected to a disordered ring-shaped geometry which is penetrated by an artificial gauge field. This particular setting is an ideal candidate for studying coherent backscattering and its interplay with atom-atom interaction beyond the mean-field approach.

The presence of very weak interaction was first accounted for in the framework of the mean-field Gross-Pitaevskii approximation. We observed an inversion of Al'tshuler-AronovSpivak oscillations, similar to the inversion of coherent backscattering [15-17]. In contrast with Refs. [15,17], a linear scaling of the inversion with $g$ was found, essentially due to the complexity of reflection and transmission amplitudes across junctions, as confirmed by nonlinear diagrammatic theory. This would imply that a further enhancement of Al'tshuler-Aronov-Spivak oscillations should be expected for weak negative $g$, provided a stationary scattering state could be realized in that case.

Truncated Wigner simulations showed that this manybody effect is subject to dephasing, in qualitative agreement with [18]. This dephasing is phenomenologically very similar to the effect of the presence of a thermal bath (for both Aharonov-Bohm [83-85] and Al'tshuler-Aronov-Spivak $[86,87]$ oscillations), the simulation of which could be carried out by very similar methodology.

More quantitatively, considering that the source injects atoms in the waveguide with a speed of $v=1 \mathrm{~mm} / \mathrm{s}$, that the $s$-wave scattering length is $a_{S}=5.313 \times 10^{-9} \mathrm{~m}$ for ${ }^{87} \mathrm{Rb}$, and that the confinement frequency is $\omega_{\perp}=2 \pi \times 1 \mathrm{kHz}$, we found that the injected atomic density would correspond to the value $\delta \rho^{\varnothing} \simeq 0.0826$. From Fig. 8 we judged that this set of parameters would correspond to a situation where a possible inversion of Al'tshuler-Aronov-Spivak oscillations would be overshadowed by dephasing, thereby giving rise to a structureless (and dominantly incoherent) transmission profile as a function of $\Phi$. Other atomic species such as ${ }^{39} \mathrm{~K}$, where the $s$-wave scattering length can be tuned to very small values [88], may be more suitable to realize an inversion of Al'tshuler-Aronov-Spivak oscillations in an experiment.

\section{ACKNOWLEDGMENTS}

The authors want to thank Thomas Wellens and Tsampikos Kottos for fruitful discussions. The computational resources have been provided by the Consortium des Equipements de Calcul Intensif (CÉCI), funded by the F.R.S.-FNRS under Grant No. 2.5020.11. J.R. acknowledges financial support from Deutsche Forschungsgemeinschaft through Project No. Ri681/14-1 and GRK 1570.

\section{APPENDIX A: DERIVATION OF THE GREEN'S FUNCTION}

We can write the total wave function of the system as

$$
\psi=P_{\mathcal{L}_{L}} \psi_{\mathcal{L}_{L}}+P_{\mathcal{R}} \psi_{\mathcal{R}}+P_{\mathcal{L}_{R}} \psi_{\mathcal{L}_{R}},
$$

with $P_{X}$ a projector onto either the left lead $\mathcal{L}_{L}$, the $\operatorname{ring} \mathcal{R}$, or the right lead $\mathcal{L}_{R}$, satisfying

$$
P_{X} P_{Y}=\delta_{X Y} P_{X}
$$

for all $X, Y=\mathcal{L}_{L}, \mathcal{R}, \mathcal{L}_{R}$. Following a scattering matrix formalism, the wave function in the left and right leads can be cast into the form

$$
\begin{aligned}
& \psi_{\mathcal{L}_{L}}=G_{\mathcal{L}_{L}} S+G_{\mathcal{L}_{L}} W_{\mathcal{L}_{L} \mathcal{R}}\left(G_{\mathcal{R}}^{-1}-\Sigma_{\mathcal{L}_{L}}-\Sigma_{R}\right)^{-1} W_{\mathcal{R} \mathcal{L}_{L}} G_{\mathcal{L}_{L}} S, \\
& \psi_{\mathcal{L}_{R}}=G_{\mathcal{L}_{R}} W_{\mathcal{L}_{R} \mathcal{R}}\left(G_{\mathcal{R}}^{-1}-\Sigma_{\mathcal{L}_{L}}-\Sigma_{\mathcal{L}_{R}}\right)^{-1} W_{\mathcal{R} \mathcal{L}_{L}} G_{\mathcal{L}_{L}} S,
\end{aligned}
$$

where we have introduced the source term $S$ and the free Green's function

$$
G_{X}=\left(\mu-H_{X}+i \epsilon\right)^{-1},
$$

with the notation $X=\mathcal{L}_{L}, \mathcal{R}, \mathcal{L}_{R}$ standing for either the left lead, the ring, or the right lead. In these equations we have also introduced the notation

$$
H_{X}=P_{X} H P_{X},
$$

which denotes the sub-Hamiltonian associated with the left lead $\mathcal{L}_{L}$, the right lead $\mathcal{L}_{R}$, or the ring $\mathcal{R}$ obtained by use of previously introduced projectors. The quantity

$$
\Sigma_{X}=W_{\mathcal{R} X} G_{X} W_{X \mathcal{R}}
$$

refers to the self-energy. The poles of a Green's function are given by the eigenenergies of the corresponding Hamiltonian. Self-energies modify these poles in a way that those also obtain an imaginary part. These new poles can be interpreted as decaying resonance states with an energy given by the real part of these poles and a lifetime associated with the imaginary part. We have also introduced the matrix elements at each junction site as

$$
W_{X \mathcal{R}}=P_{X} H P_{\mathcal{R}}=W_{\mathcal{R} X}^{\dagger},
$$


which are explicitly given by

$$
\begin{aligned}
& W_{\mathcal{L}_{L} \mathcal{R}}=-\frac{E_{\delta}}{2}|-1\rangle\langle 0|, \\
& W_{\mathcal{L}_{R} \mathcal{R}}=-\frac{E_{\delta}}{2}\left|N_{R}+1\right\rangle\left\langle\frac{N_{R}}{2}\right| .
\end{aligned}
$$

In view of first computing the Green's function of the ring, we start from the disorder and interaction free ring Hamiltonian

$H_{\mathcal{R}}=\sum_{\alpha=0}^{N_{R}-1} E_{\delta}\left(|\alpha\rangle\left\langle\alpha\left|-\frac{e^{-i \Phi}}{2}\right| \alpha\right\rangle\left\langle\alpha+1\left|-\frac{e^{i \Phi}}{2}\right| \alpha+1\right\rangle\langle\alpha|\right)$,

with $|0\rangle \equiv\left|N_{R}\right\rangle$, where $\alpha$ is an index labeling the ring sites, $N_{R}$ denotes the number of sites within the ring, $E_{\delta}$ is the onsite energy, and $\Phi$ is the artificial gauge flux. With the help of this Hamiltonian, the free 1D Green's function within the disorder-free ring lattice can be written as

$$
\begin{aligned}
\left\langle\alpha\left|G_{\mathcal{R}}\right| \alpha^{\prime}\right\rangle=\frac{1}{N_{R}} \sum_{n=0}^{N_{R}-1} \frac{e^{2 i \pi\left(\alpha-\alpha^{\prime}\right) n / N_{R}}}{\mu-\left[1-\cos \left(2 \pi n / N_{R}-\Phi\right)\right] E_{\delta}+i \epsilon} & \frac{1}{i E_{\delta} \sin k \delta}\left[e^{i\left|\alpha-\alpha^{\prime}\right|(k \delta \tilde{\Phi})} \sum_{n=0}^{\infty} e^{i n L(k \delta+\tilde{\Phi})}\right. \\
& \left.+e^{i\left(L-\left|\alpha-\alpha^{\prime}\right|\right)(k \delta-\tilde{\Phi})} \sum_{n=0}^{\infty} e^{i n L(k \delta-\tilde{\Phi})}\right],
\end{aligned}
$$

with $\tilde{\Phi}=\Phi \operatorname{sgn}\left(\alpha-\alpha^{\prime}\right)$ and $k \delta=\arccos \left(1-\mu / E_{\delta}\right)$ where the identity of Eqs. (A12) and can be shown through the application of the geometric series and Liouville's theorem. The free 1D Green's function can thus be seen as a sum over each path linking two sites. Depending on the value taken by $\alpha$ and $\alpha^{\prime}$, the path can contain several visits of each junction and many explorations of the ring arms. If one now considers smooth and weak disorder, some exponentials appearing in the above Green's function evaluation have to be rewritten as

$$
e^{i L k \delta} \rightarrow e^{i\left(\Phi_{u}+\Phi_{d}\right)},
$$

with $\Phi_{u}$ and $\Phi_{d}$ the phase due to the disorder in the upper and lower arms, respectively. One can express those phases as

$$
\begin{aligned}
\Phi_{u} & =\sum_{\alpha=0}^{N_{R} / 2-1} k_{\alpha}, \\
\Phi_{d} & =\sum_{\alpha=N_{R} / 2}^{N_{R}-1} k_{\alpha},
\end{aligned}
$$

where $k_{\alpha} \delta=\arccos \left[1-\left(\mu-V_{\alpha}\right) / E_{\delta}\right]$. We also have to make the following substitution due to the presence of disorder:

$$
e^{i\left|\alpha-\alpha^{\prime}\right| k} \rightarrow \exp \left(i \operatorname{sgn}\left(\alpha-\alpha^{\prime}\right) \sum_{\alpha^{\prime \prime}=\alpha^{\prime}}^{\alpha-1} k_{\alpha^{\prime \prime}}\right) .
$$

If we further consider the fact that the ring in the presence of disorder is coupled to the leads, we are left with the calculation of $\left[G_{\mathcal{R}}^{-1}-\left(\Sigma_{\mathcal{L}_{L}}+\Sigma_{\mathcal{L}_{R}}\right)\right]^{-1}$ that can be expanded in a perturbative series of the self-energies, hence giving rise to a Dyson series

$$
\begin{aligned}
& {\left[G_{\mathcal{R}}^{-1}-\left(\Sigma_{\mathcal{L}_{L}}+\Sigma_{\mathcal{L}_{R}}\right)\right]^{-1}} \\
& \quad=G_{\mathcal{R}}+G_{\mathcal{R}}\left(\Sigma_{\mathcal{L}_{L}}+\Sigma_{\mathcal{L}_{R}}\right) G_{\mathcal{R}} \\
& \quad+G_{\mathcal{R}}\left(\Sigma_{L}+\Sigma_{R}\right) G_{\mathcal{R}}\left(\Sigma_{\mathcal{L}_{L}}+\Sigma_{\mathcal{L}_{R}}\right) G_{\mathcal{R}}+\cdots .
\end{aligned}
$$

The total Green's function of the system is then expressed as a sum over paths within the ring with the possibility of reflection, transmission, or exit each time a junction is visited. This yields the Green's function given by Eq. (10).

\section{APPENDIX B: TECHNIQUES AND RESULTS TO CALCULATE FURTHER RESUMMED DIAGRAMS}

By solving the linear diagrammatic equation (64), we obtained the result (66). From that it is easy to derive other resummed diagrams. We obtain the diagrammatic identities and calculate

$$
\longrightarrow=e^{-i\left(\Phi_{\mathrm{u}}-\frac{\Phi}{2}\right)} \frac{r t\left(e^{2 i \Phi_{\mathrm{u}}}+e^{i\left(\Phi_{\mathrm{u}}+\Phi_{\mathrm{d}}-\Phi\right)}\right)}{D\left(\Phi_{\mathrm{u}}, \Phi_{\mathrm{d}}, \Phi\right)},
$$

Mirrored, rotated, and time-reversed versions of the already calculated diagrams can be easily derived from the latter by utilizing the following observations. 
(i) Inverting the direction of a resummed diagram is done by reverting every direction in the trajectories contained in its sum. This inversion does not change the branches which are explored, but clockwise exploration is transformed into counterclockwise exploration. The enclosed flux is thus encircled in the opposite direction, leading ultimately to a sign change of the phase $\Phi$ in the results for the diagrams.

(ii) Mirroring a diagram along the horizontal axis of the ring leads to an interchange of the upper and the lower branch of the ring and thus to an interchange $\Phi_{u} \leftrightarrow$ $\Phi_{d}$ of the associated disorder phases. Since clockwise exploration of the branches turns into counterclockwise and vice versa, we also have to change the sign of $\Phi$ again.

(iii) Mirroring a diagram along the vertical symmetry axis of the ring does not change the explored branches but interchanges clockwise and counterclockwise motion, which yet again flips the sign in front of $\Phi$.
[1] B. L. Altshuler, D. Khmel'nitzkii, A. I. Larkin, and P. A. Lee, Phys. Rev. B 22, 5142 (1980).

[2] G. Bergmann, Phys. Rep. 107, 1 (1984).

[3] P. Drude, Ann. Phys. (Leipzig) 306, 566 (1900).

[4] P. Drude, Ann. Phys. (Leipzig) 308, 369 (1900).

[5] E. Akkermans and G. Montambaux, Mesoscopic Physics of Electrons and Photons (Cambridge University Press, Cambridge, 2007).

[6] P. E. Wolf, G. Maret, E. Akkermans, and R. Maynard, J. Phys. (Paris) 49, 63 (1988).

[7] E. Akkermans, P. E. Wolf, R. Maynard, and G. Maret, J. Phys. (Paris) 49, 77 (1988).

[8] P.-E. Wolf and G. Maret, Phys. Rev. Lett. 55, 2696 (1985).

[9] B. Hapke, Icarus 157, 523 (2002).

[10] M. P. Van Albada and A. Lagendijk, Phys. Rev. Lett. 55, 2692 (1985).

[11] A. Tourin, A. Derode, P. Roux, B. A. van Tiggelen, and M. Fink, Phys. Rev. Lett. 79, 3637 (1997).

[12] J. de Rosny, A. Tourin, and M. Fink, Phys. Rev. Lett. 84, 1693 (2000).

[13] L. Margerin, M. Campillo, B. A. Van Tiggelen, and R. Hennino, Geophys. J. Int. 177, 571 (2009).

[14] F. Jendrzejewski, K. Müller, J. Richard, A. Date, T. Plisson, P. Bouyer, A. Aspect, and V. Josse, Phys. Rev. Lett. 109, 195302 (2012).

[15] M. Hartung, T. Wellens, C. A. Müller, K. Richter, and P. Schlagheck, Phys. Rev. Lett. 101, 020603 (2008).

[16] T. Wellens and B. Grémaud, Phys. Rev. Lett. 100, 033902 (2008).

[17] T. Hartmann, J. Michl, C. Petitjean, T. Wellens, J.-D. Urbina, K. Richter, and P. Schlagheck, Ann. Phys. (NY) 327, 1998 (2012).

[18] T. Geiger, A. Buchleitner, and T. Wellens, New J. Phys. 15, 115015 (2013).

[19] W. Ehrenberg and R. E. Siday, Proc. Phys. Soc. London Sect. B 62, 8 (1949).

[20] Y. Aharonov and D. Bohm, Phys. Rev. 115, 485 (1959).

[21] Y. Aharonov and D. Bohm, Phys. Rev. 123, 1511 (1961).

[22] S. Washburn and R. A. Webb, Adv. Phys. 35, 375 (1986).

[23] B. L. Altshuler, A. G. Aronov, and B. Z. Spivak, Pis'ma Zh. Eksp. Teor. Fiz. 33, 101 (1981).

[24] B. L. Al'tshuler, A. G. Aronov, and B. Z. Spivak, JETP Lett. 33, 94 (1981).

[25] D. Y. Sharvin and Y. V. Sharvin, JETP Lett. 34, 272 (1981).

[26] M. Murat, Y. Gefen, and Y. Imry, Phys. Rev. B 34, 659 (1986).

[27] A. D. Stone and Y. Imry, Phys. Rev. Lett. 56, 189 (1986).

[28] B. Douçot and R. Rammal, J. Phys. (Paris) 48, 941 (1987).
[29] B. Pannetier, J. Chaussy, R. Rammal, and P. Gandit, Phys. Rev. B 31, 3209 (1985).

[30] R. Berkovits and Y. Avishai, Phys. Rev. Lett. 76, 291 (1996).

[31] T. Wellens and B. Grémaud, J. Phys. B 39, 4719 (2006).

[32] M.-O. Mewes, M. R. Andrews, D. M. Kurn, D. S. Durfee, C. G. Townsend, and W. Ketterle, Phys. Rev. Lett. 78, 582 (1997).

[33] I. Bloch, T. W. Hänsch, and T. Esslinger, Phys. Rev. Lett. 82, 3008 (1999).

[34] V. Bolpasi, N. K. Efremidis, M. J. Morrissey, P. C. Condylis, D. Sahagun, M. Baker, and W. von Klitzing, New J. Phys. 16, 033036 (2014).

[35] G. M. Moy, J. J. Hope, and C. M. Savage, Phys. Rev. A 55, 3631 (1997).

[36] E. W. Hagley, Science 283, 1706 (1999).

[37] N. P. Robins, C. Figl, S. A. Haine, A. K. Morrison, M. Jeppesen, J. J. Hope, and J. D. Close, Phys. Rev. Lett. 96, 140403 (2006).

[38] W. Guerin, J.-F. Riou, J. P. Gaebler, V. Josse, P. Bouyer, and A. Aspect, Phys. Rev. Lett. 97, 200402 (2006).

[39] A. Couvert, M. Jeppesen, T. Kawalec, G. Reinaudi, R. Mathevet, and D. Guéry-Odelin, Europhys. Lett. 83, 50001 (2008).

[40] G. L. Gattobigio, A. Couvert, M. Jeppesen, R. Mathevet, and D. Guéry-Odelin, Phys. Rev. A 80, 041605(R) (2009).

[41] G. L. Gattobigio, A. Couvert, B. Georgeot, and D. GuéryOdelin, Phys. Rev. Lett. 107, 254104 (2011).

[42] F. Vermersch, C. M. Fabre, P. Cheiney, G. L. Gattobigio, R. Mathevet, and D. Guéry-Odelin, Phys. Rev. A 84, 043618 (2011).

[43] J. Dalibard, F. Gerbier, G. Juzeliūnas, and P. Öhberg, Rev. Mod. Phys. 83, 1523 (2011).

[44] N. Goldman, G. Juzeliūnas, P. Öhberg, and I. B. Spielman, Rep. Prog. Phys. 77, 126401 (2014).

[45] L. Amico, A. Osterloh, and F. Cataliotti, Phys. Rev. Lett. 95, 063201 (2005).

[46] A. Ramanathan, K. C. Wright, S. R. Muniz, M. Zelan, W. T. Hill, C. J. Lobb, K. Helmerson, W. D. Phillips, and G. K. Campbell, Phys. Rev. Lett. 106, 130401 (2011).

[47] T. Ernst, T. Paul, and P. Schlagheck, Phys. Rev. A 81, 013631 (2010).

[48] R. Peierls, Z. Phys. 80, 763 (1933).

[49] D. R. Hofstadter, Phys. Rev. B 14, 2239 (1976).

[50] E. Balslev and J. Combes, Commun. Math. Phys. 22, 280 (1971).

[51] S. Barry, Phys. Lett. 71A, 211 (1979).

[52] S. Barry, Ann. Math. 97, 247 (1973).

[53] B. Junker, Adv. At. Mol. Phys. 18, 207 (1982). 
[54] W. P. Reinhardt, Annu. Rev. Phys. Chem. 33, 223 (1982).

[55] Y. Ho, Phys. Rep. 99, 1 (1983).

[56] P.-O. Löwdin, Adv. Quantum Chem. 19, 87 (1988).

[57] N. Moiseyev, Phys. Rep. 302, 212 (1998).

[58] J. Dujardin, A. Argüelles, and P. Schlagheck, Phys. Rev. A 91, 033614 (2015).

[59] J.-F. Riou, Y. Le Coq, F. Impens, W. Guerin, C. J. Bordé, A. Aspect, and P. Bouyer, Phys. Rev. A 77, 033630 (2008).

[60] J. Dujardin, A. Saenz, and P. Schlagheck, Appl. Phys. B 117, 765 (2014).

[61] M. Olshanii, Phys. Rev. Lett. 81, 938 (1998).

[62] T. Paul, M. Albert, P. Schlagheck, P. Leboeuf, and N. Pavloff, Phys. Rev. A 80, 033615 (2009).

[63] J. Dujardin, T. Engl, and P. Schlagheck, Phys. Rev. A 93, 013612 (2016).

[64] T. Kottos and U. Smilansky, Phys. Rev. Lett. 79, 4794 (1997).

[65] T. Kottos and U. Smilansky, Ann. Phys. (NY) 274, 76 (1999).

[66] T. Kottos and U. Smilansky, Phys. Rev. Lett. 85, 968 (2000).

[67] P. Leboeuf and N. Pavloff, Phys. Rev. A 64, 033602 (2001).

[68] I. Carusotto, Phys. Rev. A 63, 023610 (2001).

[69] T. Paul, K. Richter, and P. Schlagheck, Phys. Rev. Lett. 94, 020404 (2005).

[70] T. Paul, P. Leboeuf, N. Pavloff, K. Richter, and P. Schlagheck, Phys. Rev. A 72, 063621 (2005).

[71] T. Paul, M. Hartung, K. Richter, and P. Schlagheck, Phys. Rev. A 76, 063605 (2007).

[72] E. H. Lieb, R. Seiringer, and J. Yngvason, Phys. Rev. A 61, 043602 (2000).

[73] T. Geiger, T. Wellens, and A. Buchleitner, Phys. Rev. Lett. 109, 030601 (2012).
[74] E. Wigner, Gruppentheorie und ihre Anwendung auf die Quantenmechanik der Atomspektren (Vieweg+Teubner, Wiesbaden, 1931).

[75] E. Wigner, Phys. Rev. 40, 749 (1932).

[76] J. E. Moyal, Proc. Cambridge Philos. Soc. 45, 99 (1949).

[77] M. J. Steel, M. K. Olsen, L. I. Plimak, P. D. Drummond, S. M. Tan, M. J. Collett, D. F. Walls, and R. Graham, Phys. Rev. A 58, 4824 (1998).

[78] A. Sinatra, C. Lobo, and Y. Castin, J. Phys. B 35, 3599 (2002).

[79] A. Polkovnikov, Phys. Rev. A 68, 053604 (2003).

[80] J. Dujardin, T. Engl, J. D. Urbina, and P. Schlagheck, Ann. Phys. (Berlin) 527, 629 (2015).

[81] T. Haug, H. Heimonen, R. Dumke, L.-C. Kwek, and L. Amico, arXiv: 1706.05180 .

[82] P. W. Anderson, Phys. Rev. 109, 1492 (1958).

[83] M. Ferrier, L. Angers, A. C. H. Rowe, S. Guéron, H. Bouchiat, C. Texier, G. Montambaux, and D. Mailly, Phys. Rev. Lett. 93, 246804 (2004).

[84] C. W. Rischau, S. Wiedmann, G. Seyfarth, D. LeBoeuf, K. Behnia, and B. Fauqué, Phys. Rev. B 95, 085206 (2017).

[85] S. Russo, J. B. Oostinga, D. Wehenkel, H. B. Heersche, S. S. Sobhani, L. M. K. Vandersypen, and A. F. Morpurgo, Phys. Rev. B 77, 085413 (2008).

[86] Y. Iye, M. Ueki, A. Endo, and S. Katsumoto, Superlatt. Microstruct. 34, 165 (2003).

[87] R. Yagi, M. Shimomura, F. Tahara, H. Kobara, and S. Fukada, J. Phys. Soc. Jpn. 81, 3707 (2012).

[88] G. Roati, C. D’Errico, L. Fallani, M. Fattori, C. Fort, M. Zaccanti, G. Modugno, M. Modugno, and M. Inguscio, Nature (London) 453, 895 (2008). 